\title{
Sarcoma Molecular Pathological Diagnosis of FFPE Tissues without Dewaxing based on Lipidomic Profiles using SpiderMass Technology
}

\section{Nina Ogrinc}

Universite de Lille Faculte des Sciences et Technologies

Pierre-Damien Caux

Université de Lille Faculté des Sciences et Technologies: Universite de Lille Faculte des Sciences et

Technologies

\section{Yves-marie Robin}

CLCC Oscar Lambret: Centre Oscar Lambret

\section{Emmanuel Bouchaert}

Oncovet Clinical Research

\section{Benoit Fatou}

Université Lille 1: Universite de Lille Faculte des Sciences et Technologies

\section{Mickael Ziskind}

Universite de Lille Faculte des Sciences et Technologies

\section{Cristian Focsa}

Université de Lille Faculté des Sciences et Technologies: Universite de Lille Faculte des Sciences et Technologies

\section{Delphine Bertin}

CLCC Oscar Lambret: Centre Oscar Lambret

\section{Dominique Tierny}

Oncovet Clinical Research

\section{Zoltan Takats}

Université de Lille Faculté des Sciences et Technologies: Universite de Lille Faculte des Sciences et Technologies

\section{Michel Salzet ( $\nabla$ michel.salzet@univ-lille.fr )}

Université de Lille: Universite de Lille https://orcid.org/0000-0003-4318-0817

\section{Isabelle Fournier}

Université de Lille Faculté des Sciences et Technologies: Universite de Lille Faculte des Sciences et Technologies

\section{Research}


Keywords: Mass Spectrometry , Water-Assisted Laser Desorption/lonization , Formalin-Fixed and Paraffin Embedded (FFPE) , Cancer diagnostic, Lipids, Metabolites, SpiderMass , Histopathology , Sarcoma

Posted Date: February 4th, 2021

DOl: https://doi.org/10.21203/rs.3.rs-157940/v1

License: (c) (i) This work is licensed under a Creative Commons Attribution 4.0 International License. Read Full License 


\section{Abstract}

Background :Formalin-fixation and paraffin-embedding (FFPE) is the worldwide gold standard for tissue preservation and routine pathology for general and cancer diagnostics. Despite robust histopathology methods, accurate diagnostic remains difficult for certain cases. Overall, the entire process is timeconsuming, labor-intensive and doesn't reach the right sensitivity and specificity. Lipids are central players in cancer, but they are not routinely used in diagnostics because of the sample processing which limits their detection, and lipid analysis directly from unprocessed FFPE tissues has never been reported.

Methods :We present a new method for cancer diagnostics based on tissue-lipidomic signatures through rapid screening of FFPE without dewaxing using Water-Assisted Laser Desorption lonization Mass Spectrometry (WALDI-MS) and deep-learning.

Results :We show on a cohort of canine and human sarcoma tissues that classification for sarcoma subtyping is possible and enables diagnosing blind samples in less than five minutes, with over $95 \%$ probability to discriminate specific subtypes.

Conclusion: We have revealed the possibility to create a rapid diagnostic platform to screen clinical FFPE tissues with minimal sample preparation for molecular pathology.

\section{Background}

Fast and accurate tumor diagnosis is critical in clinical decision making for optimal patient care, targeted therapy and disease progression monitoring. The worldwide common practice for tissue preservation is to fix the collected biopsies and surgical specimens in formalin and embed them in paraffin, aka FormalinFixed and Paraffin-Embedded (FFPE) samples. Currently, there are tens of millions of blocks stored worldwide by pathology departments and tissue banks. This is because FFPE are extremely stable, enabling indefinite storage at room temperature without degradation, and they offer well-preserved morphology for histological (HS) assessment after tissue sectioning. FFPE tissues are, thus, not only useful for high-quality histological imaging, in-situ hybridization (ISH) and immunohistochemistry (IHC), but they also represent a wealth of information for retrospective analyses. However, despite the integration of these multimodal exams, targeting both morphological and cellular tissue features as well as the distribution of specific mRNA and proteins markers, cancer diagnostics remains labor intense and difficult $(1,2)$. For example, some lesions such as soft tissue sarcomas, which form a highly complex and heterogeneous group of more than 100 known subtypes, are difficult to discriminate because a great number of them present morphological overlaps (3-5). Then, in some cases, the malignant subtypes mimic their benign counterparts (liposarcoma vs lipoma) and pose a diagnostic issue (6).

Direct mass spectrometric analysis of lipids present in tissues has gradually gained importance in course of the last 15 years(7). While originally lipid signals were perceived as interference for the detection of macromolecular species, they are now recognized as a central contributors to the pathophysiological mechanisms(8). Mass spectrometry imaging (MSI) has quickly emerged as one of the universal tools for 
studying lipid biochemistry in-situ. As the distribution and abundance of lipids follow the changes of cell phenotypes, lipid imaging has been proposed as an alternative to histology and histopathology $(9,10)$. Various methods capable of lipid MSI including Secondary lon Mass Spectrometry (SIMS), MatrixAssisted Laser Desorption Ionization (MALDI), Desorption Electrospray lonization (DESI) or Laser-Ablation Electrospray lonization (LAESI), have growing interest and were successfully applied in the clinics as molecular histology tools. In oncology, they were demonstrated for diagnostics and prognostics, cancer classification, patient stratification, and to study the tumor microenvironment(10-12). In a new approach, some methods have even been developed to enable the analysis of fresh tissue specimens or for in vivo analysis. These encompass of the Rapid Evaporative lonisation Mass Spectrometry (REIMS) which is a direct combination of an electrosurgical diathermy with on-line mass spectrometric analysis $(13,14)$; the SpiderMass minimally invasive technology that is based on micro-sampling by laser resonant excitation of the endogenous water molecules in the mid-infrared $(\lambda=2.94 \mu \mathrm{m})$ and on-line analysis by MS(1517)and the MassSpec Pen which is based on water micro-extraction of tissues and the direct analysis of the extract by MS(18).

While this uniformity of imaging and in-situ MS data is certainly advantageous in the aspect of developing a new paradigm for tissue identification, replacing cellular morphology and targeted molecular analysis, this dominance of lipids also means that the development of new methodologies will require fresh (or fresh frozen) tissue specimens. In addition to the unmatched common practice and scarce availability, the fundamental problem of fresh frozen tissue specimen is the limited stability of various lipid species. Some lipids (especially those containing polyunsaturated fatty acyl moieties) are highly sensitive to both atmospheric oxygen and UV light, making storage of tissues at room temperature under ambient atmosphere highly undesirable. However, the FFPE process is seemingly incompatible with any downstream lipidomic analysis. Overall, FFPE are more difficult to handle for molecular analysis because the paraformaldehyde reacts with various biomolecules notably by their free amine groups giving raise to Schiff-bases and further crosslinking by the formation of methylene bridges (i.e. proteins, DNA, RNA)(19). Furthermore, the analysis of the FFPE also requires the tissues to be deparaffinized and rehydrated using ethanol baths of decreasing gradients. If this doesn't represent an issue for large biomolecules that are crosslinked and very insoluble, it is expected to wash out or displace the lipid content of tissues, deeming the lipid profiling of FFPE material pointless. Yet, only protein and glycan analysis by MALDI-MSI has proven to be relevant for FFPE analysis (20) though MSI of proteins and glycans require an enzymatic digestion which lengthens the sample processing (21-25). So far, only a few groups have reported the analysis of metabolites from FFPE tissues. For example, some metabolites such as galactose-1phosphate, hexose-6-phosphate and carnitine were found by Buck et al.(26) using MALDI-MSI after deparaffinization and rehydration. Moreover, in their work Pietrowska et al.(27) have confirmed that the sample preparation steps were causing massive loss in the lipid species.

In sharp contrast with these considerations, our experimental data clearly indicates that the WaterAssisted Laser Desorption Ionization of FFPE tissue material by the SpiderMass technology without the sample preparation steps yields lipid-rich spectra and these lipid populations show excellent histological and histopathological specificity. While this discovery was absolutely unexpected, it unlocked the tens of 
millions of FFPE samples stored worldwide for lipid research and established a new pathway towards the development of a lipid profile-based paradigm in in histology.

\section{Materials And Methods Study design}

The objectives of the study were (i) to demonstrate direct analysis and classification of FFPE soft tissue tumors based on their lipido-molecular features by Water-Assisted Laser Desorption Ionization without prior sample preparation and (ii) the creation of a rapid lipido screening platform of FFPE tissues to be used in pathology labs. We first evaluated the possibility to detect lipidomic signatures directly from Adult Wistar male rat (225-250g, 7-8 weeks old) brain FFPE tissue sections collected according with European and French guidelines for animal research (European Convention for the Protection of Vertebrate Animals used for Experimental and other Scientific Purposes, ETS No.123) and approved by the local Animal Ethics committee (C2EA-075 Nord-Pas de Calais). We than investigated the lipidomolecular profiles of canine and human clinical sarcoma samples and created classification models. All dog patient samples archived at the Oncovet clinic were collected after signed owner consent. Canine sarcoma FFPE tissues blocks, archived from years 2013-2017, were selected according to four different pathologies from diverse breeds and tumor origin: Angiosarcoma (22 patients, 45 samples), Leiomyosarcoma (4 patients, 25 samples) and Fibrosarcoma (1 patients, 2 samples) and the Malignant tumor of peripheral nerve sheaths (2 patients, 4 samples). Selected surgical samples of various human soft tissue tumors fixed in 10\% formalin, embedded in paraffin and archived from years 2013-2019 in the Oscar Lambret tumor bank have been retrieved after securing patient consent: leiomyomas (4 patients, 6 samples), leiomyosarcomas ( 9 patients, 10 samples), lipomas (8 patients, 10 samples), and liposarcomas (8 patients, 11 samples). The sample size, collection and inclusion were dependent on the availability of the clinical samples from clinical databanks. The specific subtypes were selected based on the difficulty to assert correct diagnosis by the gold standard pathological workflow. The first section was stained with standard Hematoxylin-Phloxine-Saffron staining and the consecutive section was prepared for SpiderMass analysis. For histology, one veterinary pathologist (QP) Oncovet clinic read and annotated HPS canine sarcoma-stained tissues, and one human sarcoma board certified pathologist (Y-MR, Comprehensive Cancer Center Oscar Lambret Center) annotated the human sarcoma FFPE samples. The pathologists were in blind to any information about the acquisition from MS studies. No samples were excluded from the study. For the real-time analysis two blind samples of human sarcoma were randomly selected from the initial cohort.

\section{Rat Brain tissue preparations}

The 2-year-old FFPE blocks were sectioned into $8 \mu \mathrm{m}$ tissue sections using a microtome (Leica Biosystems, Wetzlar, Germany). To test the influence of the dewaxing procedure some tissue sections were dewaxed in xylene for 5 minutes in two separate jars. Then the tissue was rehydrated in descending 
order from $90 \%$ to $70 \%$ and then $30 \%$ of ethanol (5 minutes in each solution). The slide was immersed in two different solutions of $10 \mathrm{mM}$ ammonium bicarbonate (5 minutes each). The samples were dried into the desiccator prior to analyses.

\section{Histological staining and annotations}

The paraffin blocs were sliced into $7 \mu \mathrm{m}$ sections and mounted on poly-lysine coated slides. The annotations were done on Hematoxylin-Phloxine-Saffran (HPS) stained tissues. For HPS staining, a $7 \mu \mathrm{m}$ thick tissue slice consecutive to the SpiderMass-analyzed tissue was treated with hemalum solution for 1 min and rinsed with tap water. Then the tissue section was stained in Phloxine $0.1 \%$ solution for $10 \mathrm{~s}$ and rinsed with tap water before dehydration in $70 \%$ and $100 \%$ ethanol baths. Finally, the sections were dipped in saffron for $5 \mathrm{~s}$, rinsed twice in alcohol, cleaned in xylene, and mounted with cover slips and the EUKITT slide mounting medium. The stained slide was scanned for digital image acquisition using the Pannoramic MIDI slide scanner (3DHISTECH LTD. Budapest, Hungary) and the images were viewed and exported using Case viewer 1.15.

\section{Sample preparation for SpiderMass analysis}

The $7 \mu \mathrm{m}$ FFPE tissue sections of canine and human sarcoma for SpiderMass analysis were manually sprayed with a glycerol/IPA (8:2, v/v) solution in two successive passes using a sprayer (Agilent) initially designed for electrospray ionization-mass spectrometry (ESI-MS). The syringe pump (74900 series Cole Parmer Instrument Company) was set to a $300 \mu \mathrm{L} / \mathrm{min}$ flow rate. The two successive passes equal to 5 $\mu \mathrm{L}$ deposited on $1 \mathrm{~cm}^{2}$ and takes approx. $10 \mathrm{~s}$.

\section{SpiderMass instrument}

The basic design of the instrument setup is described in detail elsewhere15,16. In the following experiments, the prototype was equipped with a fibered tunable wavelength laser system between $2.8 \mu \mathrm{m}$ to $3.1 \mu \mathrm{m}$ (Radiant version 1.0.1, OPOTEK Inc., Carlsbad, USA) is pumped by the $1.064 \mu \mathrm{m}$ radiation delivered by a Q-switched 10 ns pulse width Nd:YAG laser (Quantel Laser, Les Ulis, France). The IR laser microprobe is tuned to $2.94 \mu \mathrm{m}$ to excite the most intense vibrational band of water $(\mathrm{O}-\mathrm{H})$. The major advantage of the SpiderMass system from other intraoperative mass spectrometry probes is its low invasiveness (few $\mu \mathrm{m}$ depth per shot according laser energy, 0.1-0.3 $\mathrm{mm} 3$ for a laser spot size of 400 $\mu \mathrm{m} 2)$ and the ability to screen across the sampled tissue. A 1-meter length biocompatible laser fibber with $450 \mu \mathrm{m}$ inner diameter (HP fiber, Infrared Fiber Systems, Silver Spring, USA) was connected to the exit of the OPO system and focused by a $20 \mathrm{~mm}$ focal length $\mathrm{CaF} 2$ lens attached at its end. A Tygon $\circledast$ tubing ( $2.4 \mathrm{~mm}$ inner diameter, $4 \mathrm{~mm}$ inner diameter, Akron, USA) was used to aspirate the ablated material and is directly connected to the inlet of the Q-TOF mass spectrometer (Synapt G2s for rat brain samples, and Xevo for clinical samples, Waters, Manchester) through a modified atmospheric pressure interface 
described elsewhere12. The laser energy was fixed at $4 \mathrm{~mJ} /$ pulse corresponding to a laser fluence of 3.18 $\mathrm{J} / \mathrm{cm} 2$ with variable number of laser shots.

\section{SpiderMass experiments}

The sampling position was determined based on the histopathological annotations. The samples were randomly analyzed to avoid batch effects. The acquisition sequence was composed of 10 laser shots consecutively fired between 2-4 times with a 10 s pause between each burst. Each burst results in an individual spectrum. The data was acquired in both positive and negative ion mode in sensitivity mode with $1 \mathrm{~s}$ scan time. The mass range included $\mathrm{m} / z$ 50-2000. The singly charged lipid species were predominantly observed in the $m / z 600-1000$. The Waters Xevo Q-Tof instrument has 22000 mass resolution in sensitivity mode. The acquired spectra in the figures were scaled using MassLynx software (v4.2 SNC966, Waters Laboratory Informatics). The canine and human sarcoma raw data files were than further subjected to the classification software. MS/MS spectra were recorded after the isolation of precursor ion and they were subjected to collision-induced dissociation (CID) in the transfer cell with a transfer collision energy ranging between 25 to $40 \mathrm{~V}$, depending on the selected precursor ion. The precursor ions were selected within the 1Da isolation window. The lipid annotations were performed manually through fragmentation spectra guidelines and compared to LIPIDMAPS database, Alex123 and METLIN database and literature(52-55).

\section{Statistical analysis}

The "Abstract Model Builder"- AMX (Beta, version 1.0 1972.0, Waters) software was used to build the FFPE canine and human sarcoma classification model and databank based on the recorded molecular patterns. All of the imported raw positive ion mode data were subjected to PCA and LDA-based classification models using individual spectra (on average 3 per sample). Each burst was averaged into one spectrum. The PCA analysis was used to decrease the dimensionally of the data sets and generate a list of features showing the largest variance within the data set. Further on, the features were subjected to supervised LDA through user assigned classes (sarcoma subtypes). One third of the PCs were subjected to LDA analysis. The canine classification model was built using a mass range of $600-1000 \mathrm{~m} / \mathrm{z}$ with a 0.1 binning, $1 \mathrm{e}^{3}$ threshold intensity, applied normalization and background subtraction. The human classification model was built using a mass range of $600-1000 \mathrm{~m} / \mathrm{z}$ with a 0.2 binning, $1 \mathrm{e}^{3}$ threshold intensity, applied normalization and background subtraction. The slightly larger bin size for human sarcoma samples was used to account for some small misalignments in the spectra. The performance of LDA was assessed by cross-validation. The cross-validation is performed by removing a part of the cohort and building the model again. In the experiments a " 5 -fold" and "leave-one-patient" out cross-validation were performed on the built models. Additional statistical analysis, non-parametrical one-way ANOVA (Kruskal-Wallis) followed by Dunn's test, was performed to calculate significant differences between normalized intensities for the $\mathrm{m} / \mathrm{z}$ which show the most discrimination in the LDA loading plots. 


\section{Real-time analysis}

To test the efficiency of our databank, we used "Abstract Model Builder Recognition" plugin of the "Abstract model builder" software. The analysis was performed in blind on two randomly selected samples not previously added in the classification model. The same preprocessing procedures were applied to the unclassified (test) specimens. The discriminating molecular patterns of the test specimen were extracted by means of the PCA-LDA projection matrix. The Mahalanobis distances are calculated from each previously assigned class average. The algorithm will classify the novel spectrum into the closest class, unless the distance is larger than a user defined $n *$ standard deviation of that class. In this particular experiment the outlier limit was set to 5 standard deviations. The other parameters set: $1 \mathrm{e}^{3} \mathrm{TIC}$ intensity threshold, 5 scans / spectrum, and $5 \mathrm{~s}$ timeout.

\section{Results}

\section{Optimization of WALDI-MS for analyzing unprocessed FFPE tissue sections}

Since WALDI-MS is a convenient ambient MS method for sample preparation-free tissue profiling, we have evaluated the possibility to analyze FFPE histological sections without any of the traditional sample preparation steps (i.e. the deparaffinization and the rehydration). The first assessment was performed on a FFPE rat brain tissue section which was analyzed in both positive and negative ion mode (Fig. 1). Surprisingly, a population of ions associated with the presence of lipids were detected albeit at lower intensity $\left(10 \mathrm{e}^{3}-10 \mathrm{e}^{4} \mathrm{TIC}\right.$ range) than in case of fresh-frozen tissue samples analyzed with the WALDI-MS $\left(10 e^{5}-10 e^{6} \mathrm{TIC}\right.$ range)(16). The detected species correspond to specific fatty acids ( $\left.\mathrm{m} / \mathrm{z} 200-400\right)$ and phospholipids species (m/z 700-900) in positive (Fig. 1a) as well as negative (Fig. 1b) ion mode (top panel). The signals are tissue specific since they are not detected when analyzing paraffin alone (bottom panel). Lower ion intensities can be explained by the extremely low water content of the sample which reduces the desorption/ionization efficiency of the WALDI process. Thus, in order to increase the signal intensity of the detected species, we studied the effect of adding a matrix on top of the FFPE tissues. Since a sprayed layer of water tends to shrink into a single large droplet due to low viscosity and high surface tension, we have chosen to test the addition of a thin glycerol matrix layer. Glycerol has two main advantages. First, the glycerol $\mathrm{O}-\mathrm{H}$ bond has a large absorption band that is remarkably close to the one of water (2882 vs. $2940 \mathrm{~nm}$ max absorption wavelength, respectively), and which provides important resonant excitation to generate a similar desorption/ionization process. Second, glycerol has already been successfully used as an infrared MALDI matrix in the past (28). Because of the high viscosity of glycerol, it is not possible to spray it pure and we have chosen to mix it with isopropyl alcohol (IPA). The tissues were, thus, uniformly sprayed with a $50 \%$ glycerol /50\% IPA solution. After depositing glycerol on the FFPE tissue, the lipid intensities increased by 100 -folds reaching absolute TIC intensities up to $10 \mathrm{e}^{6}$ (Fig. 1 middle panel) in both positive (Fig. 1 a middle) and negative ion mode (Fig. 1b middle). In the negative ion mode, there is a clear increase of the fatty acid species, such as m/z 255.2 Palmitic acid, 
$\mathrm{m} / \mathrm{z}=281.2$ Oleic acid, $\mathrm{m} / \mathrm{z}$ 283.2 Stearic acid and $\mathrm{m} / \mathrm{z}$ 303.2 Arachidonic acid; lipid species at $\mathrm{m} / \mathrm{z}$ 747.5 [PA (22:6_18:0)-H]-, m/z 885.5 [PI (18:0_20:4)-H]- and m/z 907.5 as well as a ganglioside GM 1 (18:0) at $\mathrm{m} / \mathrm{z}$ 1544.7. In the positive ion mode the major increase is observed for lipid species $\mathrm{m} / \mathrm{z} 734.5$ $[P C(32: 0)+H]+, 760.5[P C(34: 1)+H]+, \mathrm{m} / \mathrm{z} 782.5[\mathrm{PC}(34: 1)+\mathrm{Na}]+$ and $\mathrm{m} / \mathrm{z} 810.6[\mathrm{PC}(34: 1)+\mathrm{Na}]+$. The extensive list of MS/MS detected fragments and identified lipid species is shown in Table S1.

Furthermore, we evaluated the evolution of the MS molecular profiles and the wealth of information they contain according to the number of laser shots and time of spray. All spectra are shown in the Supplementary Information. Fig. S1 shows the mass spectra recorded in both polarities from a single laser shot. As revealed by the optical image (Fig. S1a) a partially ablated area of $0.16 \mathrm{~mm} 2$ is observed in case of the $8 \mu \mathrm{m}$ thick tissue section. Importantly, there are no significant changes in the content of the molecular profiles in either (Fig. S1b) positive or (Fig. S1C) negative ion mode for a single laser shot but a slight decrease in overall TIC intensity compared to 10 laser shots. This indicates that only a few laser shots are necessary to achieve the requested sensitivity. Next, variation of the time (1-5 min) of the glycerol deposition was tested with a single laser shot (Fig. S2 and Fig. S3). In negative mode, the overall intensity of the mass spectrum is inversely proportional to the time of spray with maximum intensity reached after the first minute of deposition (Fig. S2). In positive ion mode, however, there are only slight variations of intensities between the different times of spray (Fig. S3). To minimize the sample preparation time and improve homogeneity of the matrix layer, shorter spray times and lower viscosity of glycerol solutions were tested (data not shown) revealing that 10 s and $20 \%$ glycerol/IPA are sufficient to obtain a homogeneous layer, reproducible and high intensity signals (up to $10 \mathrm{e}^{5} \mathrm{TIC}$ ). To reinforce the importance of analyzing non-dewaxed FFPE samples, we then investigated the influence of tissue dewaxing on the detected species. After tissues were dewaxed and rehydrated according to standard protocols used in histology, mass spectra were recorded and compared to FFPE tissues analyzed without any sample treatment (Fig. 2). Dewaxed spectra (bottom) show a very different molecular profile compared to the non-dewaxed (top) with a similar trend observed in both positive (Fig. 2a) and negative (Fig. 2b) ion mode. In the dewaxed samples no signals attributed to the phospholipids were observed and only a few and low intensity signals are still found corresponding to fatty acids (e.g. signals at $\mathrm{m} / \mathrm{z} 283.2$ and 255.2). This confirms that the dewaxing procedure removes the residual lipids left behind by the dehydration process, making further lipid profiling pointless. We conclude that rapid and highly sensitive detection of lipids directly from FFPE tissue is possible using $20 \%$ glycerol in IPA and a spray time reduced down to $10 \mathrm{~s}$. Therefore, the complete process (sample preparation + analysis) requires no more than a few minutes.

\section{Establishing a rapid FFPE diagnostic screening MS-based platform using WALDI-MS}

Based on the aforementioned observations, we have created a platform for rapid lipidomic screening of FFPE tissues without deparaffinization and minimal sample preparation targeting applications in cancer diagnostics. The platform is operated in a two-stage workflow (Fig. 3). First (Fig. 3a), a molecular 
database using histologically annotated samples is constructed. The FFPE blocks from tumor tissue banks are sectioned in consecutive slides. The first section is stained and annotated by histopathology professionals; whilst the following section is analyzed by the SpiderMass system and the recorded mass spectra are subjected to data processing. First, non-supervised analysis by Principal Component Analysis (PCA) enables to check the reproducibility of the data and any potential issues associated with instrument drift or batch effects. Then, following the annotation of MS Spectra according to their histological classification, supervised analysis is performed by Linear Discriminant Analysis (LDA) to build-up classification models for the user-defined classes. Finally, these classification models are used to perform real-time screening of unknown tissues (few minutes) (Fig. 3b). The first part of the workflow (training) is crucially important for obtaining accurate results and must be achieved following well established procedures $(16,17)$. This part can take up to a few weeks (according to the number of samples, their availability and their annotation by an expert pathologist); though if tissues are available $>100$ samples can easily be analyzed per day. Then, after the training part is finalized, the identification of any sample becomes possible within $<5$ minutes using the platform.

\section{Evaluating SpiderMass FFPE analytical performances from clinical samples}

We then further evaluated the performances of the newly established methodology for the classification of real clinical cancer samples. This was achieved by analyzing a cohort of samples (29 patients; 76 samples) from canine veterinary patients with sarcoma. Canines, like humans, spontaneously develop soft tissue sarcomas, suffer from spontaneous tumor formation and mirror morphological appearances, clinico-pathological presentation, and phenotypes, which makes them an excellent comparative patients $(29,30)$. Especially, as for humans, canine sarcoma types and sub-types are numerous and the discrimination of certain sub-types represents a challenge even for an expert sarcoma pathologist. The canine FFPE classification model was constructed from the lipidomic molecular signatures, following the optimized sample preparation protocol. The collected FFPE blocs followed four main sarcoma classes from diverse breeds and tumor origin: Angiosarcoma, Leiomyosarcoma, Fibrosarcoma and Malignant tumor of peripheral nerve sheaths (Table S2). Following the collection of spectra in positive ion mode we were interested to see, if the lipido-molecular analysis of FFPE tissue would be able to discriminate between the sarcoma subtypes. The results are shown in Fig. S4. The PCA analysis (Fig. S4a) did not show any evident separation between the subtypes, however, PC2 shows a better separation between Leiomyosarcoma (red) and Angiosarcoma (grey), while, Fibrosarcoma (blue) and the Malignant tumor of peripheral nerve sheaths (violet) seem to have more similarities with angiosarcoma. The 3D-PCA model obtained reflects to intra- and inter patient heterogeneity and was in part associated with the different location of the initial tumor (e.g. skin, liver, spleen...). The selected PCAs were then subjected to supervised LDA analysis $(13,14)$ with the spectra grouped into 4-classes according to the sarcoma sub-types (Fig. S4c). A substantial discrimination is observed in LD1 from leiomyosarcoma compared to the remaining subtypes, however, they are also separating from each other in LD2 and LD3. The model gives excellent 
accuracies $99.05 \%$ using " 5 -fold" and $98.45 \%$ using "leaving-one-patient out" method (Table 1). Since some of the canine sarcoma subtypes were under-represented, we reconstructed the model including only angiosarcoma and leyiomyosarcoma (Fig. S4b) and Fig. S4d)). In this case the cross-validation accuracy slightly improved to $99.51 \%$ using "5-fold" and $99.45 \%$ using "leaving-one-patient out" method (Table 1). These first promising results show that it is possible to construct a sarcoma tissue-typing classification model based on the detected -molecular features from non-processed FFPE tissue samples.

Table 1

Cross-validation results. Cross-validation results of 4-class /2 -class dog sarcoma and 4-class/ 3-class human sarcoma models with model type, cross-validation type, $\mathrm{N}$ of classes, $\mathrm{N}$ spectra, $\mathrm{N}$ of passes as well as $\mathrm{N}$ of failures, $\mathrm{N}$ outliers and \% of correct classification accuracies after cross-validation.

\begin{tabular}{|c|c|c|c|c|c|c|c|c|}
\hline \multirow[t]{2}{*}{$\begin{array}{l}\text { Model } \\
\text { type }\end{array}$} & \multirow{2}{*}{$\begin{array}{l}\text { Cross- } \\
\text { validation } \\
\text { type }\end{array}$} & \multirow{2}{*}{$\begin{array}{l}\mathrm{N} \\
\text { classes }\end{array}$} & \multirow{2}{*}{$\begin{array}{l}\text { N } \\
\text { spectra }\end{array}$} & \multirow{2}{*}{$\begin{array}{l}\mathrm{N} \\
\text { passes }\end{array}$} & \multirow{2}{*}{$\begin{array}{l}N \\
\text { failures }\end{array}$} & \multirow[t]{2}{*}{$\begin{array}{l}\mathrm{N} \\
\text { outliers }\end{array}$} & \multicolumn{2}{|c|}{$\begin{array}{l}\text { Correct classification } \\
\text { accuracies (\%) }\end{array}$} \\
\hline & & & & & & & $\begin{array}{l}\text { Excluding } \\
\text { outliers }\end{array}$ & $\begin{array}{l}\text { Including } \\
\text { outliers }\end{array}$ \\
\hline \multirow{4}{*}{$\begin{array}{l}\text { Canine } \\
\text { sarcoma }\end{array}$} & “5-fold" & 4 & 235 & 208 & 2 & 25 & $99.05 \%$ & $88.51 \%$ \\
\hline & $\begin{array}{l}\text { "One- } \\
\text { patient- } \\
\text { out" }\end{array}$ & 4 & 235 & 191 & 3 & 41 & $98.45 \%$ & $81.28 \%$ \\
\hline & "5-fold" & 2 & 204 & 202 & 1 & 1 & $99.51 \%$ & $99.02 \%$ \\
\hline & $\begin{array}{l}\text { "One- } \\
\text { patient- } \\
\text { out" }\end{array}$ & 2 & 204 & 182 & 1 & 21 & $99.45 \%$ & $89.28 \%$ \\
\hline \multirow{4}{*}{$\begin{array}{l}\text { Human } \\
\text { sarcoma }\end{array}$} & “5-fold" & 4 & 116 & 104 & 10 & 2 & $91.23 \%$ & $89.66 \%$ \\
\hline & $\begin{array}{l}\text { "One- } \\
\text { patient- } \\
\text { out" }\end{array}$ & 4 & 116 & 78 & 28 & 10 & $73.58 \%$ & $67.24 \%$ \\
\hline & “5-fold" & 3 & 101 & 97 & 3 & 1 & $97.00 \%$ & $96.04 \%$ \\
\hline & $\begin{array}{l}\text { "One- } \\
\text { patient- } \\
\text { out" }\end{array}$ & 3 & 101 & 77 & 19 & 5 & $80.21 \%$ & $76.24 \%$ \\
\hline
\end{tabular}

\section{Histological classification of human sarcoma FFPE tissues}

In the next step, a conceptual databank was created using a cohort of human sarcoma FFPE samples (29 patients; 37 samples). Human sarcomas are known to be a very heterogeneous cancer presenting many challenges for accurate diagnostics $(31,32)$. FFPE sarcoma samples were classified (Fig. 4) based on two 2 different sarcoma types and 2 different classes (malignant and benign) (Fig. 4a). The samples were collected from patients with different age, sex, and tumor origin as shown in Table S4. Sarcomas are very rare lesions ( $1 \%$ of malignant tumors); therefore, it is difficult to obtain an equivalent number of samples of the same sub-type. The acquired spectra for each subtype are shown in Fig.S5. The PCA 
analysis (Fig. S6) shows that the first 3 PCs explain more than $80 \%$ of the total variance in the dataset (PC1 $45.5 \%$, PC2 $25.12 \%$, PC3 $14.4 \%$ ). Similarly, to canine sarcoma samples, the PCA shows a spread in the acquired data across the plane, due to intra and inter patient heterogeneity. This is particularly true for lipoma and liposarcoma FFPE samples which have a considerable number of subtypes and tumor origin (Table S3). Selected PCs were than further used for supervised LDA analysis represented by the 3D plot in Fig. 4b. A good separation is observed among the four subtypes. The first component clearly discriminates between the two sarcoma types (LD 1) while the second between the lipoma and liposarcoma cancer types (LD 2). The third (LD3) discriminates between leiomyoma and leiomyosarcoma. The loadings plot of LD2 is shown in Fig. 4c. The LD2 loadings spectra shows the most discriminatory lipid peaks (blue stars) and correspond to $\mathrm{m} / \mathrm{z} 855.8,879.8,881.8,897.8,900.8$ and 907.8. MS/MS analysis was performed in order to elucidate the identity of the selected molecular species (Table S4 and Fig. S7). Lipids contributing to the discrimination mostly correspond to a mixture of triglycerides (TG). The model gave considerably good cross-validation accuracies $91.23 \%$ using " 5 -fold" and $73.78 \%$ using "leave-one-patient out" method (Table1) for the undersized and unbalanced number of human samples. However, if we remove the under-represented leiomyoma samples and reconstruct the 3 classmodel again the cross-validation accuracies improve to $97 \%$ for " 5 -fold" and to $80 \%$ for "leaving-one patient out". In both models few benign samples were miss-classified to their malignant counterpart and vice versa. In order to improve the cross-validation accuracies and the model robustness, more samples would be needed, which is difficult for sarcoma since it is a rare disease and certain subtypes are difficult to obtain. However, further expansion of the database for clinical purposes can be achieved at any time new samples are added to the model and reprocessed.

\section{Blind screening in real-time of FFPE samples}

To demonstrate the efficiency of our platform and human sarcoma model in a clinical environment, we simulated the tissue classification in real-time. Two FFPE human sarcoma samples of different subtypes were randomly selected by the pathologist and analyzed in blind using SpiderMass accordingly to the optimized analytical workflow. The previously 4-class generated human sarcoma PCA-LDA model was loaded into AMX recognition software(13). Each acquisition is automatically predicted, and the software gives out the readout of different color-coding and percent of certainty: Leiomyoma (Red), Leiomyosarcoma (Green), Lipoma (Turquoise) and Liposarcoma (Purple). The results of the two blind acquisitions are shown in Fig. 5. The interrogation software correctly predicted in real-time with over $95 \%$ probability value of the two subtypes of human sarcoma. In the first case, the software predicted the sample to be a benign Lipoma for all three acquisitions (Fig. 5a). The scan 53-55 was predicted with $99.25 \%$ probability, scan $62-64$ with $99.28 \%$ probability and scan $71-72$ was predicted with $98.25 \%$. In the second case, the software predicted the sample to be a benign leiomyoma (red) (Fig. 5b). The prediction was with $96.21 \%$ certainty for scan $11-12$ and $97.82 \%$ probability for scan $23-24$, and finally $95.38 \%$ for scan 33-34. Following the fast screening, the results were compared to the histological annotations of the pathologist confirming the correct classification in both cases obtained in real-time with the SpiderMass 
platform. These results show it is possible to provide correct classification accuracy for blind sample, above $95 \%$, already from a rather small training cohort of samples.

\section{Effect of FFPE conservation time on molecular profiles}

A particularly important aspect of the analysis of FFPE samples is to study the dependence of molecular profiles on the FFPE protocol as well as on the age of the samples. It is known that FFPE tissues undergo chemical changes with the conservation time. In order to check how this can affect our analysis, we investigated the effects of the embedding as well as age of the sample on the lipid profiles. In the first example, a recently embedded (2019) human lipoma sample was analyzed before and after sample preparation (Fig. 6a and Fig. 6b). The mass spectra following the deposition of $20 \%$ glycerol in IPA spray show a significant increase $(\mathrm{x} 100)$ in signal intensities of some lipid species mainly assigned as the previously identified TG (52:4) / TG(50:1) Na+ at m/z 855.8, TG (54:6) / TG(52:3) Na+ at m/z 879.8, TG (54:5) / TG(52:2) Na+ at m/z 881.8 and TG (56:6) / TG(54:3)Na+ at m/z 907.8. For comparison we also added a spectrum of an un-treated fresh-frozen human lipoma sample (Fig. $6 \mathrm{c}$ ). While most lipid peaks between $\mathrm{m} / \mathrm{z}$ 800-950 are retained in FFPE tissue, several lipids in the mass range $\mathrm{m} / \mathrm{z}$ 700-800 are not observed after fixation and paraffin embedding. The identity of the $\mathrm{m} / \mathrm{z} 881.8$ species was confirmed in fresh-frozen and FFPE tissue by MS/MS analysis (Fig. 7). In the second example, we performed a sample age dependence study, where we acquired MS spectra from Leiomyosarcoma samples at different years of embedding from 2014-2019. The results are shown in Fig. 8. First, we compared the lipid profiles obtained from the year 2014, 2015 and 2019 of embedding Fig. 8a. There is a clear drop in intensity observed when comparing the lipid profiles. In the lipid mass range $\mathrm{m} / \mathrm{z} 600-900$ the absolute intensities of the peaks in 2014 and 2015 are only about $10 \%$ of the value in 2019 (Fig. S8). Furthermore, PCA analysis of the collected samples (Fig. 8b) was performed to get an overview of the impact of conservation time. The PC 1 describes $62.27 \%$ variance; PC 2 describes $12.47 \%$ and PC $311.29 \%$. There is a progressive separation of the data along PC 1 from the 2019 to a bundle at 2014, 2015 and 2016 with 2016 and 2017 in between. The loading-mass plot of PC1 indicated 4 most significant peaks which contribute to the variance $\mathrm{m} / \mathrm{z} 643.6,853.8,879.8$ and 881.8 (data not shown). Statistical tests were performed on the normalized intensities for each of peak and expressed as box plots with Tukey whisker definition Fig. 8c. The data show there is a significant difference between the intensities of the $\mathrm{m} / \mathrm{z} 853.8$, 879.8 and 881.8 between the samples embedded 2016 and 2019 and only m/z 881.8 between the 2015 and 2019. No significant difference is observed between the embedding years 2014 and 2019. The statistical tests, therefore, indicated that only a small fraction, the 3 of the most variant peaks (in the year 2016), show a significant difference. However, differences are observed, with losses of absolute intensities for certain signals, when analyzing the material embedded after a period ( $\max 3$ years).

\section{Discussion}

We report for the first time a direct lipid profiling technique of FFPE tissues with the SpiderMass requiring minimal sample pre-processing. Because the method does not require the tissue sections to be 
deparaffinized and rehydrated but only a fast spray (deposition of matrix, $\leq 1 \mathrm{~min}$ ), neither costly equipment nor well trained staff is necessary to operate the platform and the effective screening of blind samples can be performed in less than $5 \mathrm{~min}$. This rapid analysis meets the requirements of the clinical post-operative turn-over time because the number of samples that are transiting everyday by the pathology service at the hospital require the analytical process to be as short as possible. We have shown that histologically different molecular features were obtained and used to classify clinical samples. Interestingly, soft tissue tumor subtypes known to be difficult to distinguish morphologically by the pathologist (lipoma versus well-differentiated liposarcoma or liposarcoma lipoma-like) were successfully separated by using the molecular fingerprints recorded with the platform. Annotation is central to associate the area analyzed from a tissue to its correct class defining the ground truth. For sarcoma, the samples were classified based on their subtypes; angiosarcoma, leiomyosarcoma, fibrosarcoma and malignant tumor of peripheral nerve sheaths for canine patients; and leiomyoma, leiomyosarcoma, lipoma and liposarcoma for human patients. A similar approach has already been conducted in a study, where the SpiderMass system was used to analyze fresh-frozen canine sarcoma biopsies ex vivo allowing for correct classification (97\% of specificity) of tumor type and grading (17). In our case, the dog and human models based on sarcoma subtyping gave good classification accuracies $99 \%$ and $91 \%$, respectively, with " 5 -fold" cross-validation method and $99 \%$ and $73 \%$, respectively, with the "leaving-one patient out" approach (Table 1). The majority of misclassification in human sarcomas was due to the spectra being identified as malignant instead of the benign tissue of the same sarcoma subtype. This reflects to the fact that these specific subtypes (lipoma/liposarcoma) are morphologically alike and can be misidentified by the pathologist. Although the "leaving-one-patient-out" $(16,33,34)$ method is the reference cross-validation technique, it also produces errors when dealing with under-represented classes. This is well exemplified in our experiments with the human sarcoma for which under-represented number of samples are available for certain less prevalent sarcoma subtypes. This is particularly true for leiomyoma, where only 3 patients could be included in the model. Once the model is rebuilt for only 3 human sarcoma subtypes with removed leiomyoma samples, the cross-validation with " 5 -fold" improves to $97 \%$, while the "leave-one patient out" to $80 \%$. Despite the lower "leave-one patient out" cross-validation accuracies of the model the real-time interrogation of two blind samples resulted in correct classification accuracy above $95 \%$ for leiomyoma and above $98 \%$ for lipoma (Fig. 5). This demonstrates that the pipeline for rapid FFPE screening can be easily setup for pathology diagnosis. The classification models can be setup according to the considered cancer type taking histological and morphological features (normal, tumor, necrosis), grades, types (and sub-types) or stages into account. It is of great importance in the learning phase to target a balanced and well represented number of samples for each class of the model. The time to build the classification models only depends on the availability of the necessary tissues and their annotation by a pathologist, because more than 100 specimens can be analyzed per day.

In general, FFPE tissues analysis for cancer diagnostics is challenging due to the chemical modifications of endogenous biomolecules during the whole sample processing workflow (initial fixation, dehydration, paraffin embedding, and then deparaffinization and rehydration when tissues are analyzed). Despite

Page 14/32 
chemical modifications induced already by the formalin fixation, successful protocols were established to assess protein and DNA/RNA molecular content. Indeed, DNA and RNA sequencing is possible with nextgeneration sequencing and PCR protocols $(35,36)$. For targeted protein analysis with IHC, antigen retrieval (AR) such as Heat Induced Antigen Retrieval (HIAR) have been long -established to unmask the epitope which is masked by the network of the crosslinks between different molecules. Alongside, strategies were developed to perform proteomics by AR combined with enzymatic digestion (most often trypsin) from FFPE $(37,38)$. Protocols have been optimized over the years and nowadays thousands of proteins can be identified from archived tissues (39). In 2007, strategies were also proposed to unlock mass spectrometry imaging (MSI) of proteins from FFPE tissues (21) and nowadays protocols for protein analysis from FFPE tissue are highly reproducible (23). However, small molecule analysis raises different issues. Due to their solubility and molecular weight, lipids and metabolites are more sensitive to the tissue processing. It has already been demonstrated a long time ago(40) that certain class of lipids were undergoing degradation upon formaldehyde fixation. For example, in the brain, phosphatidylcholines(PC), phosphatidylethanolamine (PE) and phosphatidylserine (PE) were found to be broken down to their corresponding lyso compounds, fatty acids, and phosphatidic acids. This was more recently investigated using high-performance LC-MS from archived hospital formalin-fixed (FF) samples as well as lipid standards incubated with formalin. In the study by Gaudin et al. (41) phospholipids (PL) were shown to undergo hydrolysis or modifications ( $\mathrm{N}$-methylation and $\mathrm{N}$-formylation for phospholipids bearing an amine) whilst the sphingolipids (SL) were not affected by the fixation. MALDI MSI from FF rat brain tissues was also successfully investigated (42). These observations were recently confirmed from FF tissues of mouse kidney (43) from which PL levels were observed to be decreased compared to unfixed tissues. If FF samples present certain chemical modifications; FFPE samples are even more complex to analyze due to fixation, dehydration, paraffin embedding, deparaffinization and rehydration steps prior to the analysis. These induce "the washing out" of many lipids from the tissues as previously shown (27). However, in 2011, Kelly et al. (44) developed a protocol for metabolite extractions from FFPE samples using $80 \% \mathrm{MeOH}$ combined with $30-45 \mathrm{~min}$ heating at $70^{\circ} \mathrm{C}$ followed by cooling to congeal the wax. Despite the well-thought processing, only few metabolites could be identified but a good reproducibility (> 95\% for all except one sample) was obtained from repeated analysis of archived sarcoma tissues. Recently, a complete study from cells and tissues with comparison of unfixed, FF and FFPE samples was conducted by Cacciatore and coworkers using the same protocol(45). They showed that only approximatively $40-50 \%$ lipids and metabolites could be retrieved and in particular glycerophospholipids were hardly obtained. It has also been demonstrated, that despite the formalin fixation and paraffin embedding it is possible to retain some metabolites to enable MALDI MS imaging of FFPE tissues(46, 47). However, the sample preparation in these experiments is time-consuming and requiring on-hands expertise while not providing real-time analysis. On the other hand, we have demonstrated that it is possible to obtain lipid information directly from the FFPE tissues with no removal of the paraffin and minimal pre-processing of the tissue followed by real-time MS analyses. Since our platform doesn't necessitate deparaffinization and rehydration, many phospholipids species such as PAs, PSs, PIs and PCs were observed and identified from FFPE rat brain tissues in positive and negative ion mode as well as a 
mixture of Triglycerides (TGs) in Human Sarcoma FFPE tissue sections in positive ion mode. These lipid species were used as classifiers to build the molecular databases.

The IR laser microprobe of the SpiderMass system is tuned to excite the most intense vibrational band of water $(\mathrm{O}-\mathrm{H})$ which acts as an endogenous matrix. Since, FFPE tissues do not retain water, an exogenous matrix is necessary to induce the desorption/ionization process. This is clearly observed by the low signal intensities in the mass spectra without sample preparation in both rat brain and sarcoma (Fig. 1, Fig. 6). Glycerol structure contains $(\mathrm{O}-\mathrm{H})$ bonds like water which promote desorption/ionization with the IR SpiderMass laser. This effect is observed by signal intensities of some lipid species reaching $100 x$ the intensity of the initial measurement in FFPE brain tissue analysis (Fig. 1) similar to what is usually obtained by SpiderMass from fresh-frozen tissues (16). The exact same effect is observed when measuring the FFPE preserved human sarcoma tissues in positive ion mode (Fig. 6). Two successive layers of the $20 \%$ glycerol/IPA (approx. 10 s spray) have a tremendous impact on lipid signal intensity. The improved sensitivity of lipid signals enabled us to construct classification databanks from canine and human sarcoma subtypes.

Human sarcomas are a rare and complex type of cancer forming more than 100 different histopathological subtypes. They form a class of highly heterogeneous mesenchymal lesions which can arise from fat, smooth and striated muscle, cartilage, bone, fibrous connective tissue, and the pericytes of small blood vessels in the subcutis and from skin(31) Current diagnostic methods include basic analytical approaches such as standard histological staining, immunostaining and molecular genetics $(32,48)$. Without these ancillary testing methods the first-hand morphological diagnostic approach can be extremely challenging particularly for tumors of intermediate malignant potential. For example, certain benign lesions may, due to their high cellularity mimic low grade bland-looking sarcomas. This is particularly true for lipoma-like liposarcoma which is morphologically almost identical6 to lipoma. By providing new sarcoma classification models based on lipid molecular profiles we can help pathologist assert precise diagnosis. The developed histology-level classification models showed a molecular discrimination between the four subtypes of sarcoma as well as malignant vs. benign types. The discrimination between the lipoma and liposarcoma was due to specific Triglyceride lipid species identified by the MS/MS analysis (Table S4 and Fig. S7). The most prevalent triglycerides TG (52:4) / TG(50:1) Na + at m/z 855.8, TG (54:5) / TG(52:2) Na + at m/z 881.8 and TG (56:6) / TG(54:3) Na + at m/z 907.8 found in benign lipomas have been previously found to decrease in fresh-frozen high-grade myxoid liposarcomas by MALDI-MSI(49). Triglycerides are an independent source for fatty-acid oxidation which governs cell proliferation and tumor growth(50). This has strong implications why these species are more prevalent in benign lipoma than malignant liposarcoma. However, further studies would need to be conducted in order to validate these markers. Several lipid species were observed depleted between the fresh-frozen and recently fixed and paraffin embedded lipoma sample. Though most discriminative TGs remained untouched, a portion of lipids in the mass range between $\mathrm{m} / \mathrm{z} 700-800$ were depleted in FFPE lipoma. 
In our study, we also investigated the effect sample age on the lipid profiles. It is known that crosslinking processes continue to progress in the FFPE blocks for years and that the older the blocks gets, the tighter are the crosslinked networks of molecules inside the block. The block is thus becoming a highly hydrophobic entity demonstrated by the impossibility to dissolve the tissue at room temperature. For proteomics, that can be compensated by using HIAR which helps with the extraction and digestion of proteins. However, differences were observed in the identification by large scale proteomic approaches of older samples (loss of less abundant proteins)(51). For lipids we also observed an effect of the conservation and time of conservation on the detection of the lipid species from FFPE. A drop of absolute lipid signal intensities was observed when comparing fresh-frozen lipoma to FFPE lipoma in the $\mathrm{m} / \mathrm{z}$ range between 600-1000, mostly likely corresponding to phospholipids. Hydrolysis and depletion of phospholipids was similarly observed to previously reported data from FF tissues(41). However, hydrolysis is not expected to increase with the conservation time. Thus, we hypothesize that the decrease of intensities arises from the increase of crosslinking in the older samples. However, no time dependent study was previously performed from FFPE in order to systematically investigate the effect of conservation time on lipids analysis. Nevertheless, despite the decrease of certain signals, statistical tests indicated that only a small fraction, the 3 of the most variant peaks, show a significant difference.

\section{Conclusion}

To conclude, we have revealed the possibility to create a rapid diagnostic SpiderMass platform to screen clinical FFPE tissues with minimal sample preparation. The speed of the screening procedure is exemplified in the videos conducted during the unknown sample evaluation (Video 1, Video 2). The creation of canine and human FFPE sarcoma databanks are just one of the examples where the platform could be used for consolidation of histopathological diagnosis. Tissue banks with different types of FFPE cancer could utilize this platform. However, any further implementation into the clinical environment will require further elaboration of the database as well as on-site validation with larger sample cohorts of the technique. Moreover, we determined, that samples up to 6 years old, can be used, to create a databank increasing our availability of large sample cohorts. The speed of the sample preparation and fast acquisition (less than $5 \mathrm{~min}$ ) clearly demonstrates the great potential of this SpiderMass platform to be used in every pathological laboratory. Future automation of the platform can be envisioned to provide pathologist with a new, fast and robust tool for diagnostic that can be easily inserted in their routine process. Finally, deployment of the platform for FFPE lipids MS imaging is also something that we wish to further explore that is of high interest due to the minimal sample preparation involved compared to conventional MALDI.

\section{Abbreviations}

DESI : Desorption Electrospray lonization

FFPE : Formalin-fixation and paraffin-embedding 
HPS : Hematoxylin-Phloxine-Saffran

(HS) : Histological.

IHC : immunohistochemistry

ISH : in-situ hybridization

LAESI : Laser-Ablation Electrospray lonization

MALDI : Matrix-Assisted Laser Desorption Ionization

$\mathrm{MSI}$ : Mass spectrometry imaging

SISM : Secondary lon Mass Spectrometry

REIMS : Rapid Evaporative Ionisation Mass Spectrometry

WALDI-MS : Water-Assisted Laser Desorption Ionization Mass Spectrometry

\section{Declarations}

\section{Ethics approval and consent to participate :}

All experiments were approved by the local Ethics Committee (CPP Nord Ouest IV 12/10) in accordance with the French and European legislation. Prior to the experiments, patients signed an informed consent and authorization form describing the experimental protocol. No personal information was used in these experiments, and a random number was assigned to each sample.

\section{Consent for publication :}

All patient consent for publication

Availability of data and material : All raw data has been deposited to the Mendeley Data repository and will be accessible with a code prior to publication.

\section{Competing interests:}

N.O., P-D.C., Y-M.R., D.B., B.F., E.B declare they have no competing interests. M.Z, C.F., M.S. and I.F. are inventors on a patent (priority number WO2015IB57301 20150922) related to part of the described protocol. E.B. is an employee of OCR. D.T. is founder and CEO of OCR. The system is under protection by patent CA2961491 A1 (29). 
This work was funded by Ministère de l'Enseignement Supérieur, de la Recherche et de l'Innovation, Université de Lille and Inserm. The project was also funded by Institut Universitaire de France (I.F.) and Région Hauts de France-EU FEDER O'DREAMS (D.T., I.F., M.S.). ISite ULNE, Region Haut de France-EU FEDER and OCR funded the PhD of P-D.C.

\section{Author contributions :}

N.O. wrote the manuscript original draft; N.O and I.F. designed the experiments; N.O., P-D.C., and B.F performed the experiments. E.B. and D.T. collected the canine sarcoma samples and performed the histology. Y-M.R. and D.B. collected the human sarcoma samples, performed histology and validated diagnostics. N.O., P-D.C. and B.F. analyzed the data. I.F., M.S. Y-M.R. and Z.T. corrected the manuscript. I.F. and M.S. supervised the project and; M.Z., C.F., M.S. and I.F., provided the funding.

\section{Acknowledgments:}

wish to thank Marc Le Parquier from the "Centre d'Études et de Recherches, Lasers et Applications" (CERLA) of the University of Lille for his technical support. We also thank all the members of SATT-Nord involved in SpiderMass, and in particular, Eglantine Rollet and François-Xavier Denimal, for their support to the project.

\section{References}

1. S. Joseph, R. Janakiraman, G. Chacko, R. Jayaraj, M. Thomas, M. Thomas, S. Mukhopadhyay, Predictability of Recurrence using Immunohistochemistry to delineate Surgical Margins in mucosal Head and Neck Squamous Cell Carcinoma (PRISM-HNSCC): study protocol for a prospective, observational and bilateral study in Australia and India. BMJ Open. 7, e014824 (2017).

2. M. Mino-Kenudson, L. R. Chirieac, K. Law, J. L. Hornick, N. Lindeman, E. J. Mark, D. W. Cohen, B. E. Johnson, P. A. Jänne, A. J. lafrate, S. J. Rodig, A novel, highly sensitive antibody allows for the routine detection of ALK-rearranged lung adenocarcinomas by standard immunohistochemistry. Clin. Cancer Res. 16, 1561-1571 (2010).

3. T. Mentzel, G. Palmedo, C. Kuhnen, Well-differentiated spindle cell liposarcoma ('atypical spindle cell lipomatous tumor') does not belong to the spectrum of atypical lipomatous tumor but has a close relationship to spindle cell lipoma: clinicopathologic, immunohistochemical, and molecular analysis of six cases. Modern Pathology. 23, 729-736 (2010).

4. A. Deyrup, F. Chibon, L. Guillou, P. Lagarde, J.-M. Coindre, S. Weiss, Fibrosarcoma-like Lipomatous Neoplasm: A Reappraisal of So-called Spindle Cell Liposarcoma Defining a Unique Lipomatous Tumor Unrelated to Other Liposarcomas. The American Journal of Surgical Pathology. 37, 13731378 (2013). 
5. WHO Classification of Tumours of Soft Tissue and Bone. Fourth Edition - WHO - OMS -, (available at https://apps.who.int/bookorders/anglais/detart1.jsp?codlan=1\&codcol=70\&codcch=4005).

6. J. G. Azzopardi, J. Iocco, R. Salm, Pleomorphic lipoma: a tumour simulating liposarcoma. Histopathology. 7, 511-523 (1983).

7. X. Han, Lipidomics for studying metabolism. Nature Reviews Endocrinology. 12, 668-679 (2016).

8. M. L. Sulciner, A. Gartung, M. M. Gilligan, C. N. Serhan, D. Panigrahy, Targeting lipid mediators in cancer biology. Cancer Metastasis Rev. 37, 557- 572 (2018).

9. D. Touboul, A. Brunelle, O. Laprévote, Mass spectrometry imaging: Towards a lipid microscope? Biochimie. 93, 113-119 (2011).

10. R. Longuespée, R. Casadonte, M. Kriegsmann, C. Pottier, G. P. de Muller, P. Delvenne, J. Kriegsmann, E. D. Pauw, MALDI mass spectrometry imaging: A cutting-edge tool for fundamental and clinical histopathology. PROTEOMICS - Clinical Applications. 10, 701-719 (2016).

11. J. Quanico, J. Franck, M. Wisztorski, M. Salzet, I. Fournier, in Neuroproteomics: Methods and Protocols, F. H. Kobeissy, Jr. Stevens Stanley M., Eds. (Springer, New York, NY, 2017; https://doi.org/10.1007/978-1-4939-6952-4_2), Methods in Molecular Biology, pp. 21-43.

12. R. J. DeHoog, J. Zhang, E. Alore, J. Q. Lin, W. Yu, S. Woody, C. Almendariz, M. Lin, A. F. Engelsman, S. B. Sidhu, R. Tibshirani, J. Suliburk, L. S. Eberlin, Preoperative metabolic classification of thyroid nodules using mass spectrometry imaging of fine-needle aspiration biopsies. PNAS. 116, $21401-$ 21408 (2019).

13. J. Balog, L. Sasi-Szabó, J. Kinross, M. R. Lewis, L. J. Muirhead, K. Veselkov, R. Mirnezami, B. Dezső, L. Damjanovich, A. Darzi, J. K. Nicholson, Z. Takáts, Intraoperative Tissue Identification Using Rapid Evaporative Ionization Mass Spectrometry. Science Translational Medicine. 5, 194ra93-194ra93 (2013).

14. K.-C. Schäfer, J. Dénes, K. Albrecht, T. Szaniszló, J. Balog, R. Skoumal, M. Katona, M. Tóth, L. Balogh, Z. Takáts, In Vivo, In Situ Tissue Analysis Using Rapid Evaporative Ionization Mass Spectrometry. Angewandte Chemie International Edition. 48, 8240-8242 (2009).

15. B. Fatou, P. Saudemont, E. Leblanc, D. Vinatier, V. Mesdag, M. Wisztorski, C. Focsa, M. Salzet, M. Ziskind, I. Fournier, In vivo Real-Time Mass Spectrometry for Guided Surgery Application. Sci Rep. 6, 25919 (2016).

16. N. Ogrinc, P. Saudemont, J. Balog, Y.-M. Robin, J.-P. Gimeno, Q. Pascal, D. Tierny, Z. Takats, M. Salzet, I. Fournier, Water-assisted laser desorption/ionization mass spectrometry for minimally invasive in vivo and real-time surface analysis using SpiderMass. Nat Protoc. 14, 3162-3182 (2019).

17. P. Saudemont, J. Quanico, Y.-M. Robin, A. Baud, J. Balog, B. Fatou, D. Tierny, Q. Pascal, K. Minier, M. Pottier, C. Focsa, M. Ziskind, Z. Takats, M. Salzet, I. Fournier, Real-Time Molecular Diagnosis of Tumors Using Water-Assisted Laser Desorption/Ionization Mass Spectrometry Technology. Cancer Cell (2018), doi:10.1016/j.ccell.2018.09.009.

18. J. Zhang, J. Rector, J. Q. Lin, J. H. Young, M. Sans, N. Katta, N. Giese, W. Yu, C. Nagi, J. Suliburk, J. Liu, A. Bensussan, R. J. DeHoog, K. Y. Garza, B. Ludolph, A. G. Sorace, A. Syed, A. Zahedivash, T. E. 
Milner, L. S. Eberlin, Nondestructive tissue analysis for ex vivo and in vivo cancer diagnosis using a handheld mass spectrometry system. Science Translational Medicine. 9, eaan3968 (2017).

19. H. Puchtler, S. N. Meloan, On the chemistry of formaldehyde fixation and its effects on immunohistochemical reactions. Histochemistry. 82, 201-204 (1985).

20. R. Longuespée, C. Boyon, A. Desmons, D. Vinatier, E. Leblanc, I. Farré, M. Wisztorski, K. Ly, F. D’Anjou, R. Day, I. Fournier, M. Salzet, Ovarian cancer molecular pathology. Cancer Metastasis Rev. 31, 713732 (2012).

21. R. Lemaire, A. Desmons, J. C. Tabet, R. Day, M. Salzet, I. Fournier, Direct Analysis and MALDI Imaging of Formalin-Fixed, Paraffin-Embedded Tissue Sections. J. Proteome Res. 6, 1295-1305 (2007).

22. M. Wisztorski, J. Franck, M. Salzet, I. Fournier, in Mass Spectrometry Imaging: Principles and Protocols, S. S. Rubakhin, J. V. Sweedler, Eds. (Humana Press, Totowa, NJ, 2010; https://doi.org/10.1007/978-1-60761-746-4_18), Methods in Molecular Biology, pp. 303-322.

23. A. M. Judd, D. B. Gutierrez, J. L. Moore, N. H. Patterson, J. Yang, C. E. Romer, J. L. Norris, R. M. Caprioli, A recommended and verified procedure for in situ tryptic digestion of formalin-fixed paraffinembedded tissues for analysis by matrix-assisted laser desorption/ionization imaging mass spectrometry. Journal of Mass Spectrometry. 54, 716-727 (2019).

24. R. R. Drake, C. A. West, A. S. Mehta, P. M. Angel, in Glycobiophysics, Y. Yamaguchi, K. Kato, Eds. (Springer, Singapore, 2018; https://doi.org/10.1007/978-981-13-2158-0_4), Advances in Experimental Medicine and Biology, pp. 59-76.

25. T. W. Powers, B. A. Neely, Y. Shao, H. Tang, D. A. Troyer, A. S. Mehta, B. B. Haab, R. R. Drake, MALDI Imaging Mass Spectrometry Profiling of N-Glycans in Formalin-Fixed Paraffin Embedded Clinical Tissue Blocks and Tissue Microarrays. PLOS ONE. 9, e106255 (2014).

26. A. Buck, A. Ly, B. Balluff, N. Sun, K. Gorzolka, A. Feuchtinger, K.-P. Janssen, P. J. Kuppen, C. J. van de Velde, G. Weirich, F. Erlmeier, R. Langer, M. Aubele, H. Zitzelsberger, M. Aichler, A. Walch, Highresolution MALDI-FT-ICR MS imaging for the analysis of metabolites from formalin-fixed, paraffinembedded clinical tissue samples. The Journal of Pathology. 237, 123-132 (2015).

27. M. Pietrowska, M. Gawin, J. Polańska, P. Widłak, Tissue fixed with formalin and processed without paraffin embedding is suitable for imaging of both peptides and lipids by MALDI-IMS. PROTEOMICS. 16, 1670-1677 (2016).

28. A. Overberg, M. Karas, U. Bahr, R. Kaufmann, F. Hillenkamp, Matrix-assisted infrared-laser $(2.94 \mu \mathrm{m})$ desorption/ionization mass spectrometry of large biomolecules. Rapid Communications in Mass Spectrometry. 4, 293-296 (1990).

29. F. Sultan, B. A. Ganaie, Comparative oncology: Integrating human and veterinary medicine. Open Vet J. 8, 25-34 (2018).

30. W. Liu, M. Bennett, T. Helm, Canine melanoma: a comparison with human pigmented epithelioid melanocytoma. International Journal of Dermatology. 50, 1542-1545 (2011).

31. J. S. Bleloch, R. D. Ballim, S. Kimani, J. Parkes, E. Panieri, T. Willmer, S. Prince, Managing sarcoma: where have we come from and where are we going?: Therapeutic Advances in Medical Oncology 
(2017), doi:10.1177/1758834017728927.

32. C. R. Antonescu, The role of genetic testing in soft tissue sarcoma. Histopathology. 48, 13-21 (2006).

33. D. L. Phelps, J. Balog, L. F. Gildea, Z. Bodai, A. Savage, M. A. El-Bahrawy, A. V. Speller, F. Rosini, H. Kudo, J. S. McKenzie, R. Brown, Z. Takáts, S. Ghaem-Maghami, The surgical intelligent knife distinguishes normal, borderline and malignant gynaecological tissues using rapid evaporative ionisation mass spectrometry (REIMS). Br. J. Cancer. 118, 1349-1358 (2018).

34. E. R. St John, J. Balog, J. S. McKenzie, M. Rossi, A. Covington, L. Muirhead, Z. Bodai, F. Rosini, A. V. M. Speller, S. Shousha, R. Ramakrishnan, A. Darzi, Z. Takats, D. R. Leff, Rapid evaporative ionisation mass spectrometry of electrosurgical vapours for the identification of breast pathology: towards an intelligent knife for breast cancer surgery. Breast Cancer Research. 19, 59 (2017).

35. S. S. Auerbach, D. P. Phadke, D. Mav, S. Holmgren, Y. Gao, B. Xie, J. H. Shin, R. R. Shah, B. A. Merrick, R. R. Tice, RNA-Seq-based toxicogenomic assessment of fresh frozen and formalin-fixed tissues yields similar mechanistic insights. Journal of Applied Toxicology. 35, 766-780 (2015).

36. C. Heydt, J. Fassunke, H. Künstlinger, M. A. Ihle, K. König, L. C. Heukamp, H.-U. Schildhaus, M. Odenthal, R. Büttner, S. Merkelbach-Bruse, Comparison of Pre-Analytical FFPE Sample Preparation Methods and Their Impact on Massively Parallel Sequencing in Routine Diagnostics. PLOS ONE. 9, e104566 (2014).

37. P. D. Piehowski, V. A. Petyuk, R. L. Sontag, M. A. Gritsenko, K. K. Weitz, T. L. Fillmore, J. Moon, H. Makhlouf, R. F. Chuaqui, E. S. Boja, H. Rodriguez, J. S. H. Lee, R. D. Smith, D. M. Carrick, T. Liu, K. D. Rodland, Residual tissue repositories as a resource for population-based cancer proteomic studies. Clinical Proteomics. 15, 26 (2018).

38. B. L. Hood, M. M. Darfler, T. G. Guiel, B. Furusato, D. A. Lucas, B. R. Ringeisen, I. A. Sesterhenn, T. P. Conrads, T. D. Veenstra, D. B. Krizman, Proteomic analysis of formalin fixed prostate cancer tissue. Molecular \& Cellular Proteomics (2005), doi:10.1074/mcp.M500102-MCP200.

39. F. Coscia, S. Doll, J. M. Bech, L. Schweizer, A. Mund, E. Lengyel, J. Lindebjerg, G. I. Madsen, J. M. Moreira, M. Mann, A streamlined mass spectrometry-based proteomics workflow for large-scale FFPE tissue analysis. The Journal of Pathology. 251, 100-112 (2020).

40. F. A. DEIERKAUF, F. J. M. HESLINGA, THE ACTION OF FORMALDEHYDE ON RAT BRAIN LIPIDS: Journal of Histochemistry \& Cytochemistry (2017), doi:10.1177/10.1.79.

41. M. Gaudin, M. Panchal, S. Ayciriex, E. Werner, A. Brunelle, D. Touboul, C. Boursier-Neyret, N. Auzeil, B. Walther, C. Duyckaerts, O. Laprévote, Ultra performance liquid chromatography - mass spectrometry studies of formalin-induced alterations of human brain lipidome. Journal of Mass Spectrometry. 49, 1035-1042 (2014).

42. C. L. Carter, C. W. McLeod, J. Bunch, Imaging of Phospholipids in Formalin Fixed Rat Brain Sections by Matrix Assisted Laser Desorption/Ionization Mass Spectrometry. J. Am. Soc. Mass Spectrom. 22, 1991 (2011). 
43. D. R. N. Vos, A. P. Bowman, R. M. A. Heeren, B. Balluff, S. R. Ellis, Class-specific depletion of lipid ion signals in tissues upon formalin fixation. International Journal of Mass Spectrometry. 446, 116212 (2019).

44. A. D. Kelly, S. B. Breitkopf, M. Yuan, J. Goldsmith, D. Spentzos, J. M. Asara, Metabolomic Profiling from Formalin-Fixed, Paraffin-Embedded Tumor Tissue Using Targeted LC/MS/MS: Application in Sarcoma. PLOS ONE. 6, e25357 (2011).

45. S. Cacciatore, G. Zadra, C. Bango, K. L. Penney, S. Tyekucheva, O. Yanes, M. Loda, Metabolic Profiling in Formalin-Fixed and Paraffin-Embedded Prostate Cancer Tissues. Mol Cancer Res (2017), doi:10.1158/1541-7786.MCR-16-0262.

46. A. Ly, A. Buck, B. Balluff, N. Sun, K. Gorzolka, A. Feuchtinger, K.-P. Janssen, P. J. K. Kuppen, C. J. H. van de Velde, G. Weirich, F. Erlmeier, R. Langer, M. Aubele, H. Zitzelsberger, L. McDonnell, M. Aichler, A. Walch, High-mass-resolution MALDI mass spectrometry imaging of metabolites from formalin-fixed paraffin-embedded tissue. Nature Protocols. 11, 1428-1443 (2016).

47. A. Buck, B. Heijs, B. Beine, J. Schepers, A. Cassese, R. M. A. Heeren, L. A. McDonnell, C. Henkel, A. Walch, B. Balluff, Round robin study of formalin-fixed paraffin-embedded tissues in mass spectrometry imaging. Anal Bioanal Chem. 410, 5969-5980 (2018).

48. O. I. Jaber, P. A. Kirby, Alveolar Soft Part Sarcoma. Arch. Pathol. Lab. Med. 139, 1459-1462 (2015).

49. S. M. Willems, A. van Remoortere, R. van Zeijl, A. M. Deelder, L. A. McDonnell, P. C. Hogendoorn, Imaging mass spectrometry of myxoid sarcomas identifies proteins and lipids specific to tumour type and grade, and reveals biochemical intratumour heterogeneity. The Journal of Pathology. 222, 400-409 (2010).

50. S. Balaban, L. S. Lee, M. Schreuder, A. J. Hoy, Obesity and cancer progression: is there a role of fatty acid metabolism? Biomed Res Int. 2015, 274585 (2015).

51. B. Donczo, A. Guttman, Biomedical analysis of formalin-fixed, paraffin-embedded tissue samples: The Holy Grail for molecular diagnostics. Journal of Pharmaceutical and Biomedical Analysis. 155, 125-134 (2018).

52. M. Sud, E. Fahy, D. Cotter, A. Brown, E. A. Dennis, C. K. Glass, A. H. Merrill, R. C. Murphy, C. R. H. Raetz, D. W. Russell, S. Subramaniam, LMSD: LIPID MAPS structure database. Nucleic Acids Res. 35, D527D532 (2007).

53. E. Fahy, S. Subramaniam, R. C. Murphy, M. Nishijima, C. R. H. Raetz, T. Shimizu, F. Spener, G. van Meer, M. J. O. Wakelam, E. A. Dennis, Update of the LIPID MAPS comprehensive classification system for lipids. J Lipid Res. 50, S9-S14 (2009).

54. J. K. Pauling, M. Hermansson, J. Hartler, K. Christiansen, S. F. Gallego, B. Peng, R. Ahrends, C. S. Ejsing, Proposal for a common nomenclature for fragment ions in mass spectra of lipids. PLOS ONE. 12, e0188394 (2017).

55. Smith, G. O’Maille, E. J. Want, C. Qin, S. A. Trauger, T. R. Brandon, D. E. Custodio, R. Abagyan, G. Siuzdak, METLIN: a metabolite mass spectral database. Ther Drug Monit. 27 (2005). 


\section{Figures}

a)

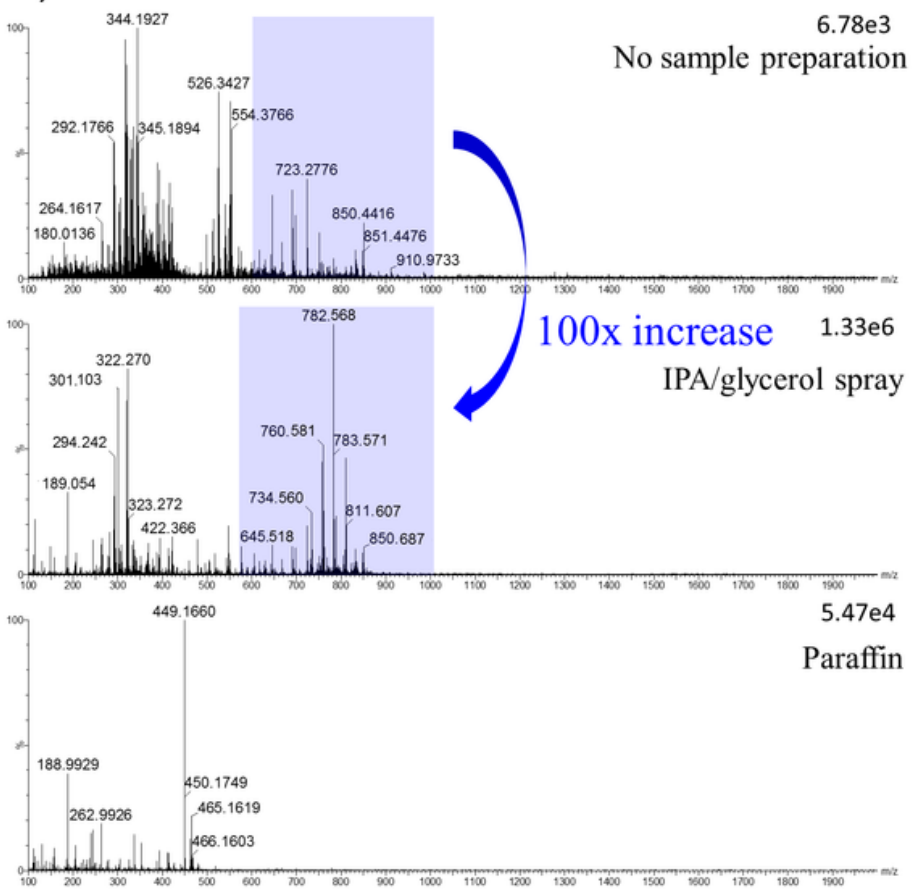

b)

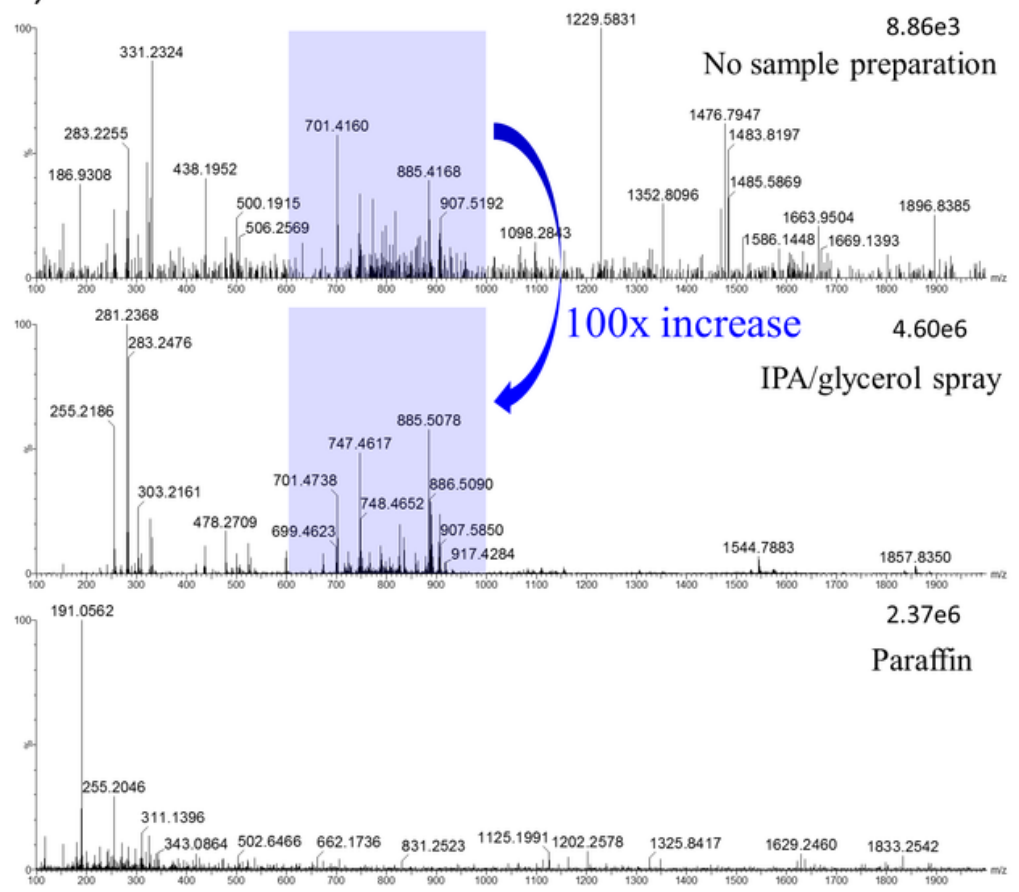

\section{Figure 1}

FFPE brain tissue MS spectra. SpiderMass acquired a) positive and b) negative ion mode MS spectra of FFPE brain tissue sections. The top spectra were acquired without the sample preparation, the middle with the 2 passes of $50 \%$ IPA/glycerol spray and the bottom spectra corresponds to the paraffin. The highlighted (blue box) lipid mass range $\mathrm{m} / \mathrm{z} 600-1000$ indicates the signal intensity increase by $100 \mathrm{x}$ between the sample without the sample preparation and the sprayed slide 
a)
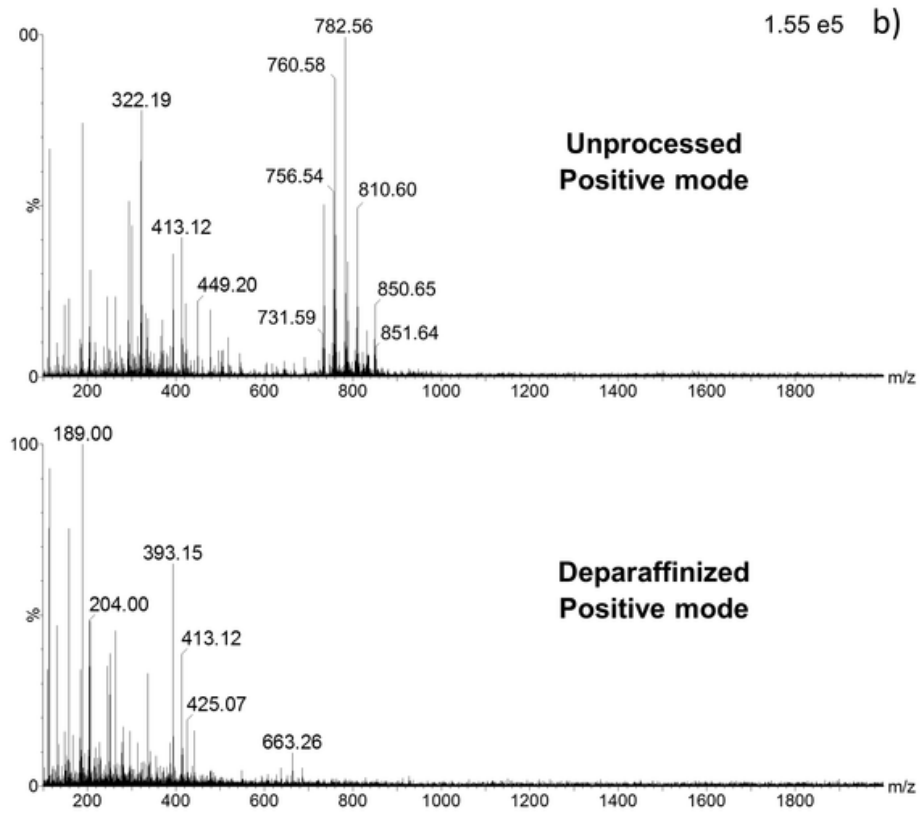
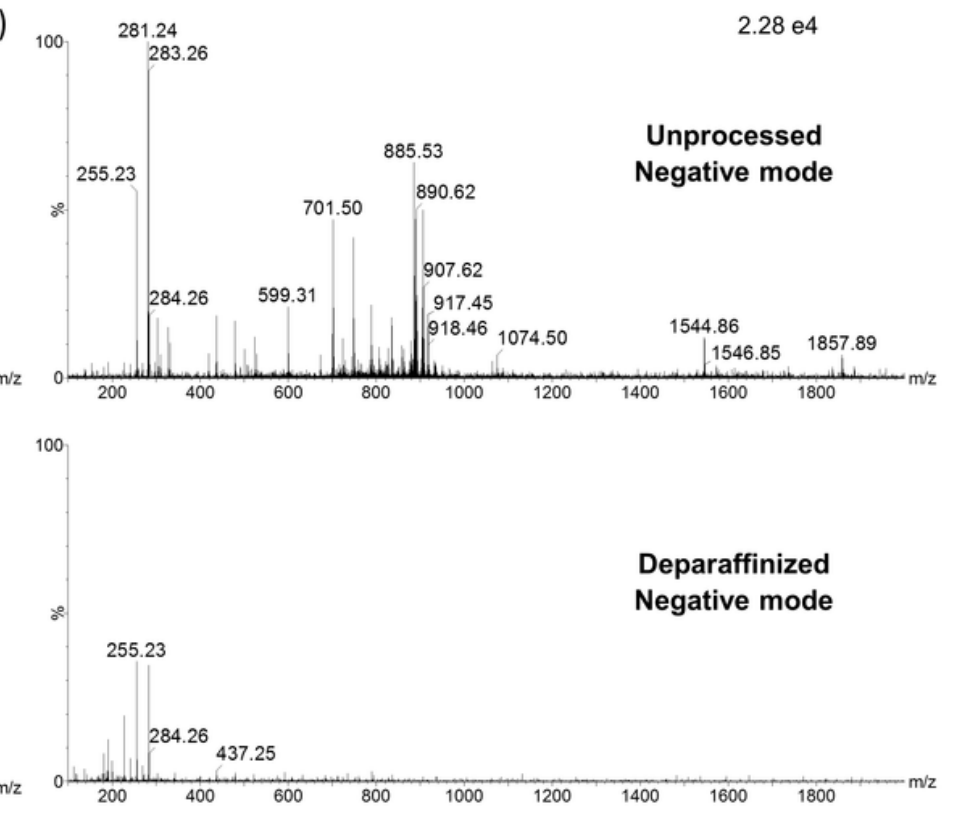

\section{Figure 2}

FFPE brain tissue MS spectra after dewaxing. in a) positive ion mode and b) negative ion mode. All of the spectra intensities have been scaled. Comparison of FFPE rat brain tissue without (top spectra) and with deparaffinization (bottom spectra). The time of spray of IPA/glycerol was 1 minute. The spectra were acquired at $4 \mathrm{~mJ} /$ pulse and in a duration of one laser shot. 
a)

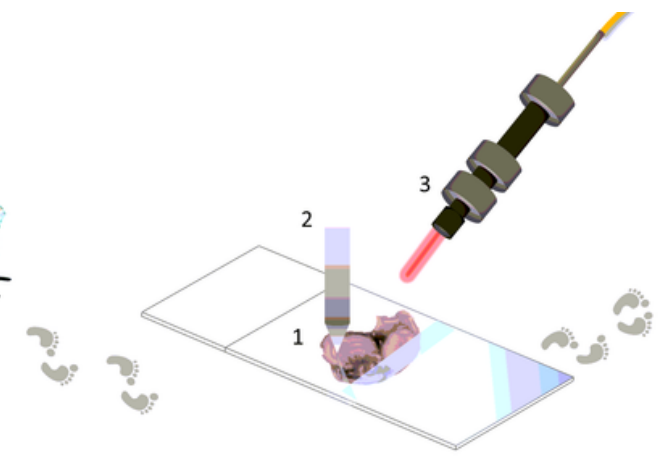

Histopathological annotations

SpiderMass analysis
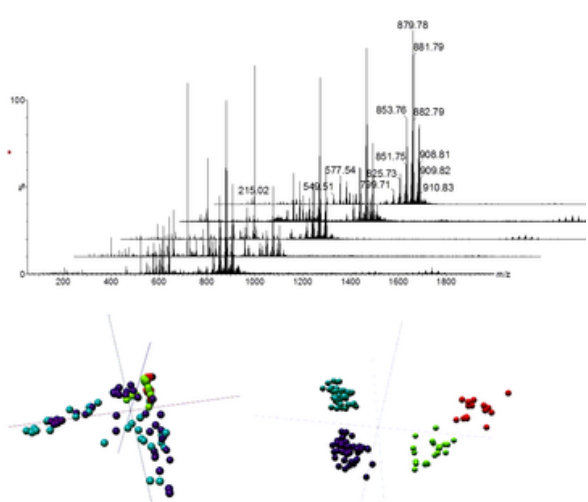

Data collection and processing

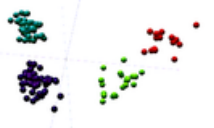

b)

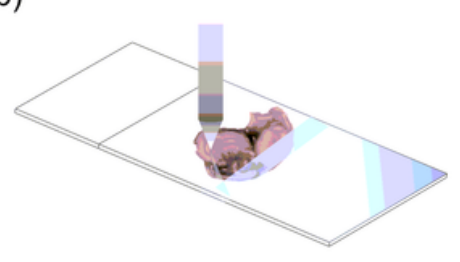

$10 \mathrm{~s}$

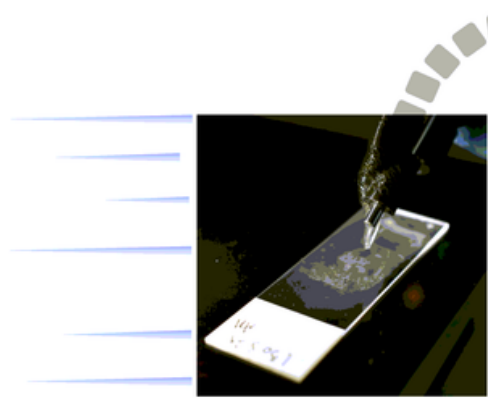

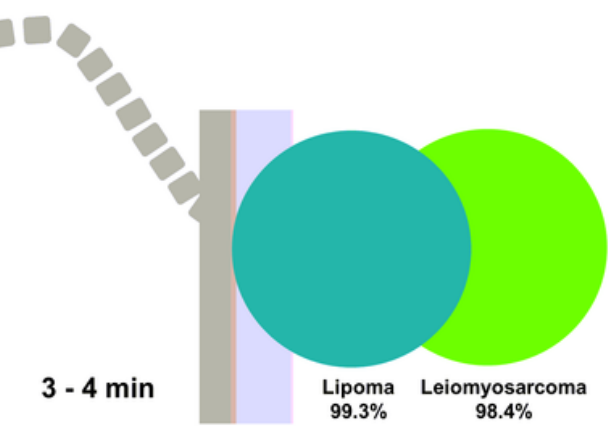


a)

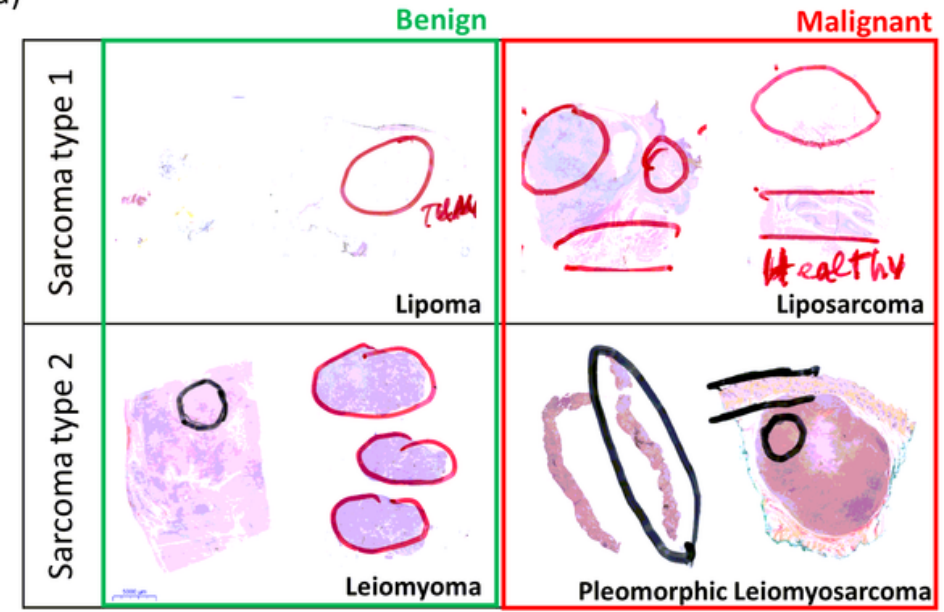

b)

LD 3

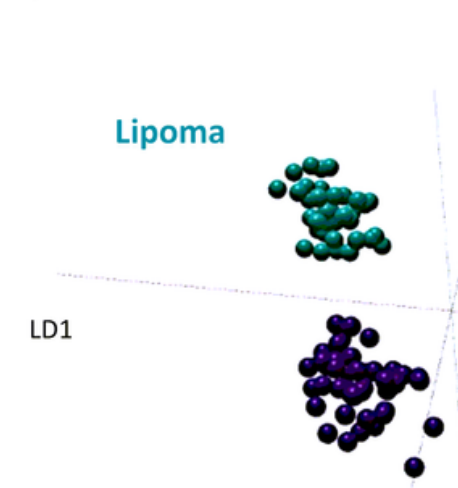

Liposarcoma

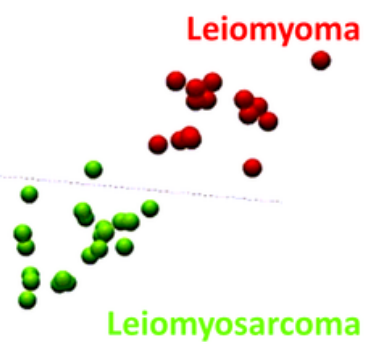

LD2

c)

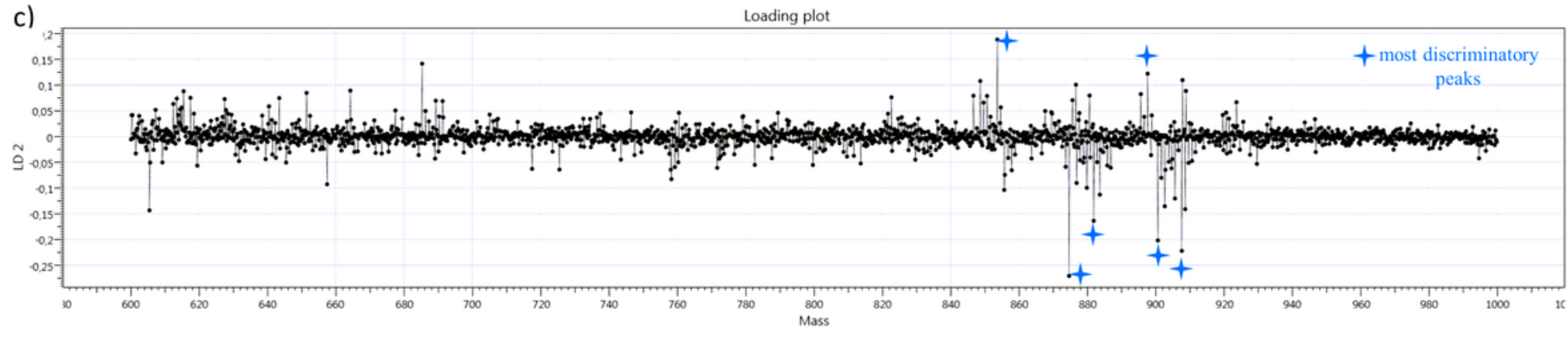

Figure 4

Building the PCA-LDA classification model based on 4 Human Sarcoma types (Lipoma, Liposarcoma, Leiomyoma or Leiomyosarcoma). A) Examples of histopathological stains and annotations of FFPE sarcoma samples. The table separates them based on their type (Sarcoma type 1-lipoma, liposarcoma and Sarcoma type 2- leiomyoma and leiomyosarcoma) and based on tumor classification: benign (green box) or malignant (red box). B) LDA representation of the 4-class PCA-LDA. The LD1 discriminates between the two sarcoma types. The model was built from the individual spectra recorded in positive ion mode. C) LD1 loading plot showing the discrimination between the benign (green) and malignant (red) tumors. The blue stars indicate the most discriminatory peaks: m/z 855.8, 879.8, 881.8, 897.8, 900.8 and 907.8 . 
a)

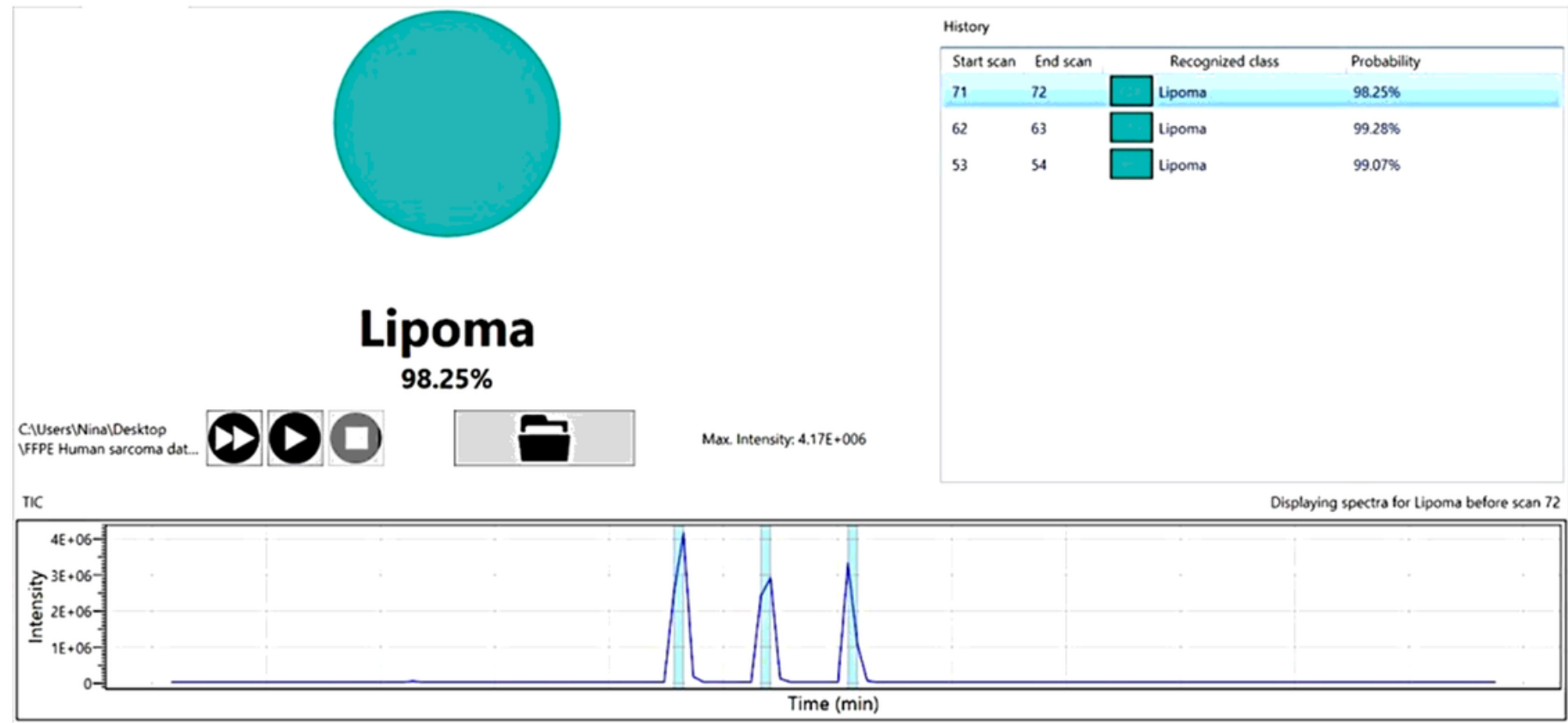

b)

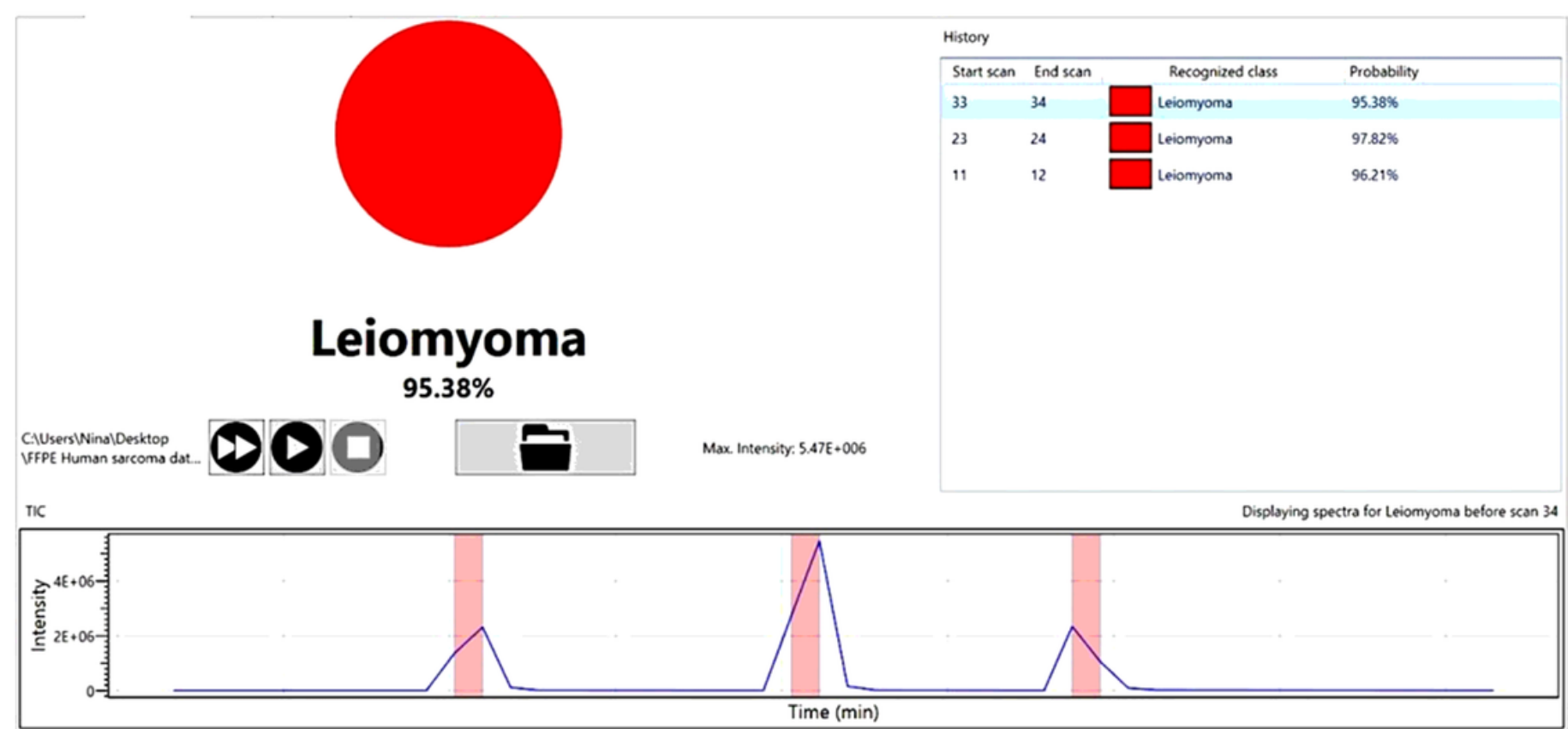

Figure 5

Real-time interrogation of the built classification model with 2 blind samples a) lipoma (turquoise) and b) leiomyoma (red). The interrogation software correctly predicted Scan 53-55 with $99.25 \%$ probability, Scan 62-64 with $99.28 \%$ probability and Scan $71-72$ with $98.25 \%$ for Lipoma. In the second case, the software predicted the sample to be a benign leiomyoma (red) with $96.21 \%$ certainty for Scan 11-12 and $97.82 \%$ probability for Scan 23-24, and finally $95.38 \%$ for Scan 33-34. 
a)

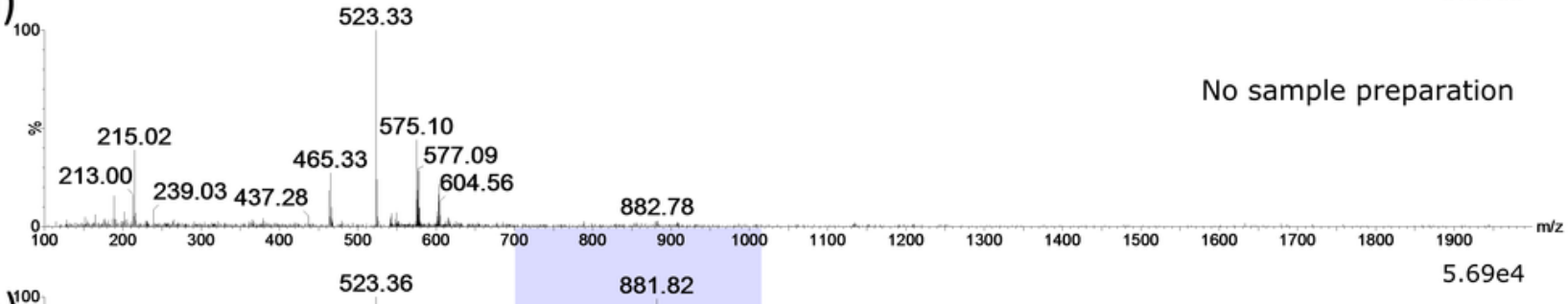

b)

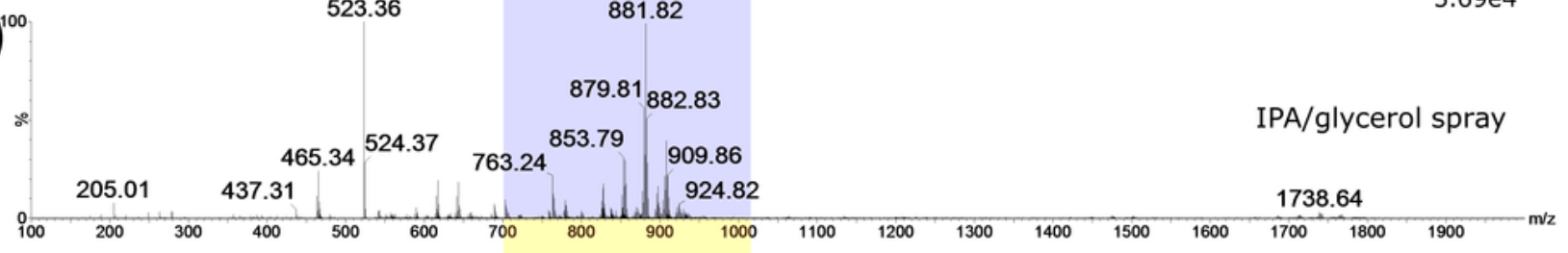

c)
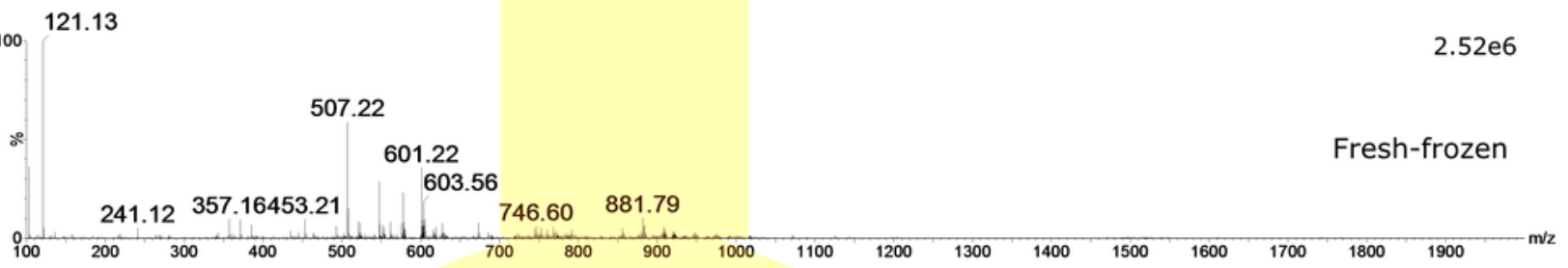

d)

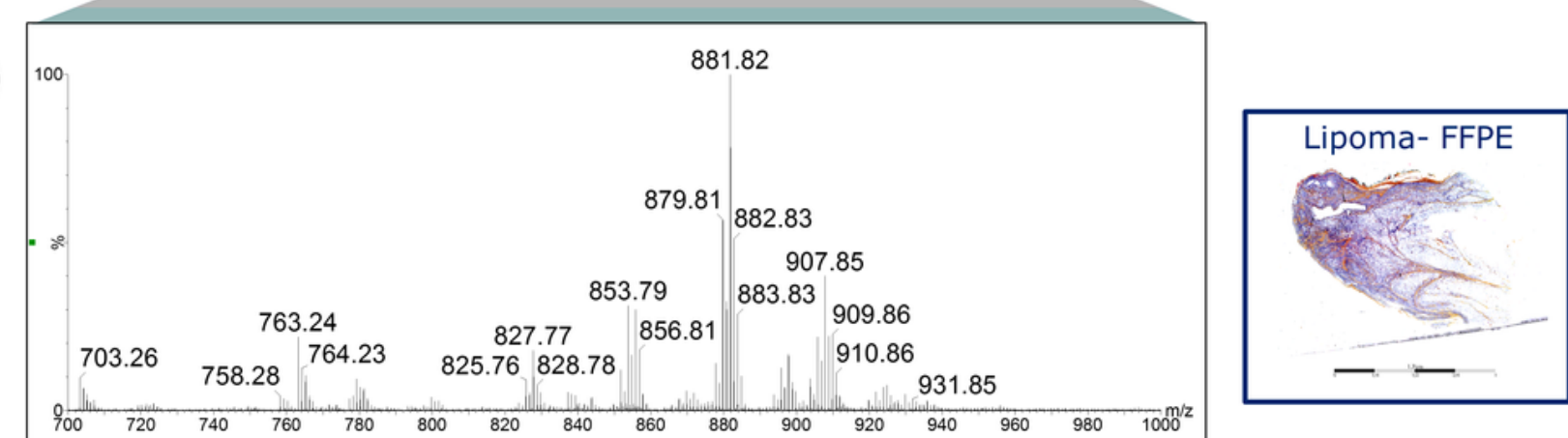

e)
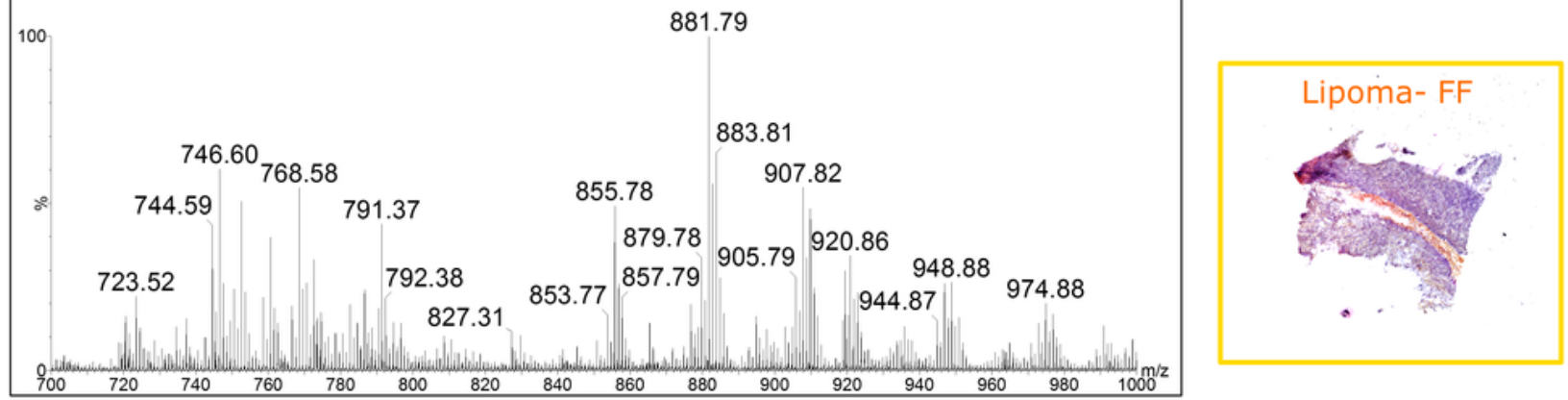

Figure 6

Acquired spectra of the FFPE and Fresh-Frozen Lipoma tissue in positive ion mode. The FFPE tissue sections of lipoma a) without sample preparation and with b) 10s glycerol/IPA spray. FFPE lipoma molecular profile was compared with c) fresh-frozen lipoma molecular profile without sample preparation. d) \& e) correspond to zoom in the $\mathrm{m} / \mathrm{z}$ region [700-1000] of MS spectra presented in c) and d) respectively as well as HPS optical scans of the analyzed tissue sections. 


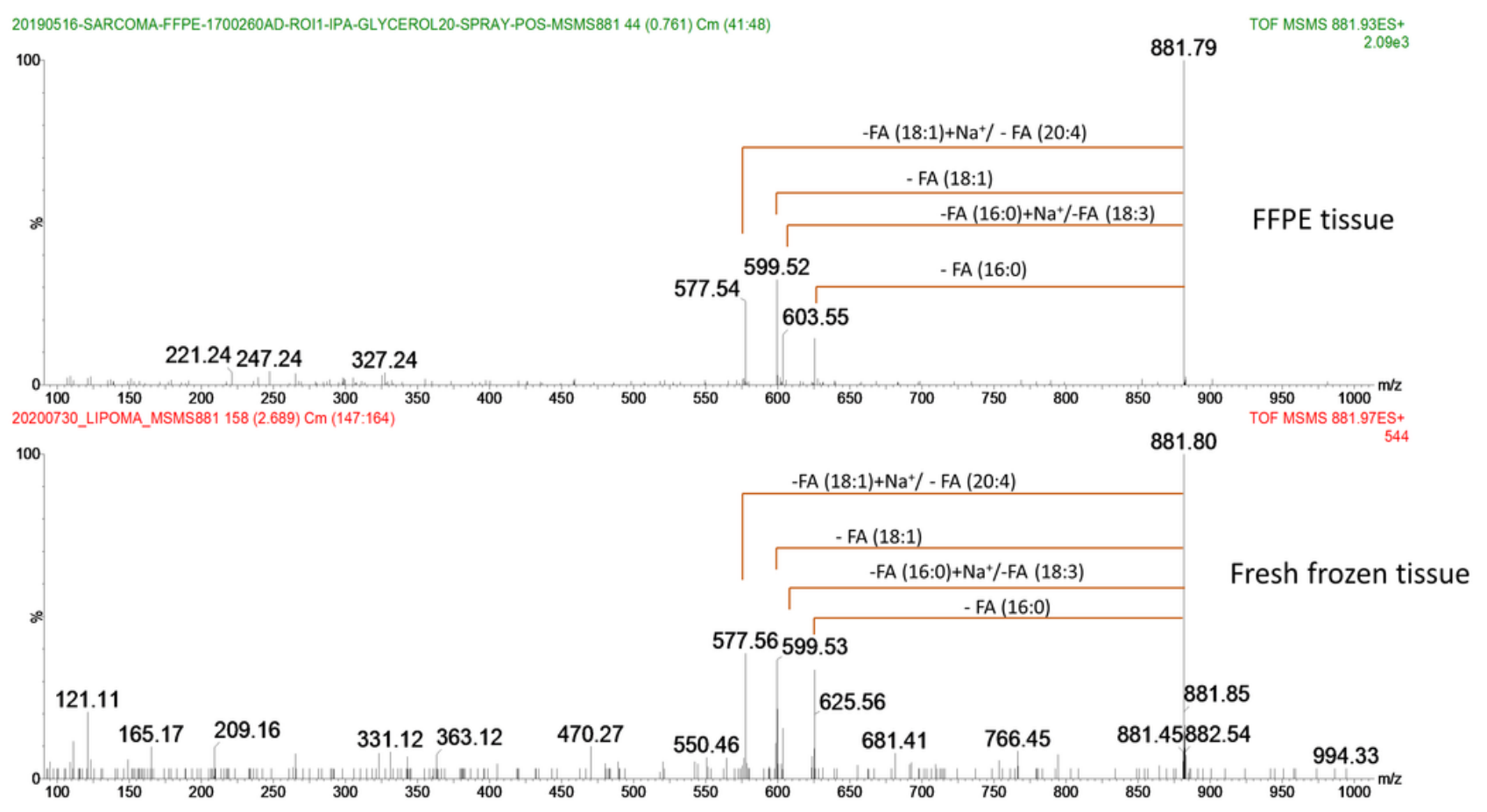

\section{Figure 7}

Positive ion mode MS/MS spectra of the TG (54:5)/TG (52:2) Na+. The species was selected at $\mathrm{m} / \mathrm{z}$ 881.8 in human sarcoma tissue. Spectrum from FFPE tissue(top) and fresh-frozen tissue (bottom). The CID energy was set to $35 \mathrm{eV}$. The isolation window was set to $1 \mathrm{Da}$. 
a.
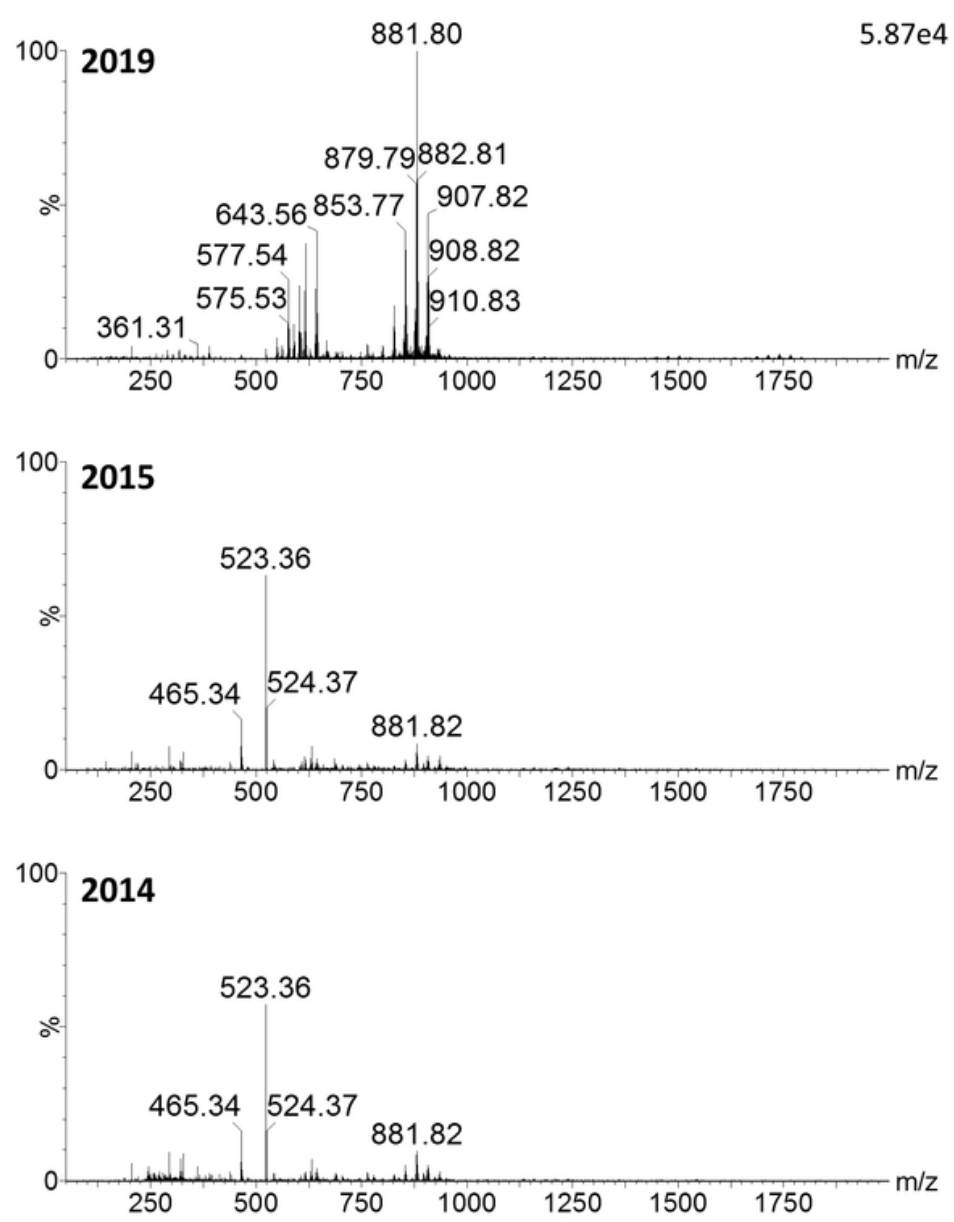

b.

PC3
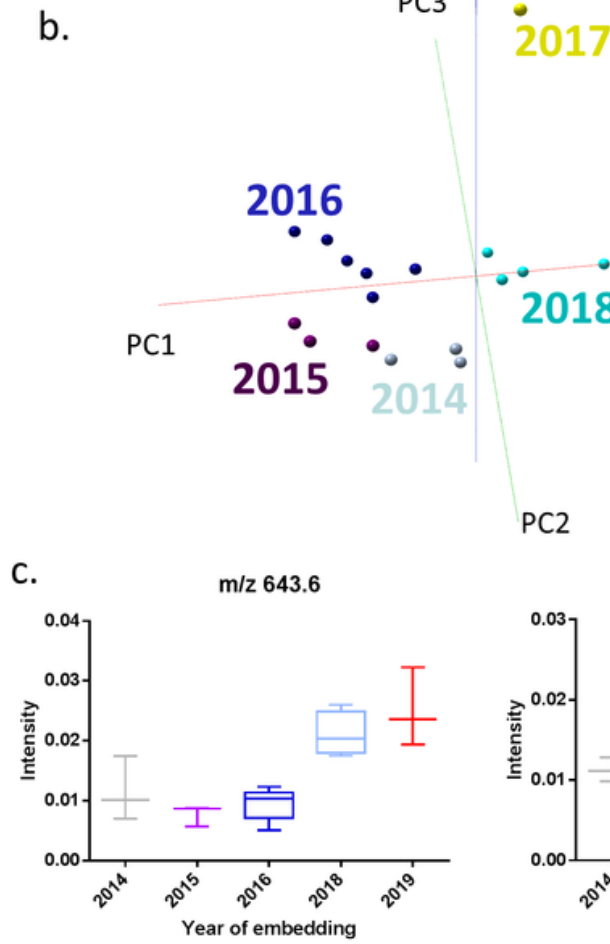

PC2
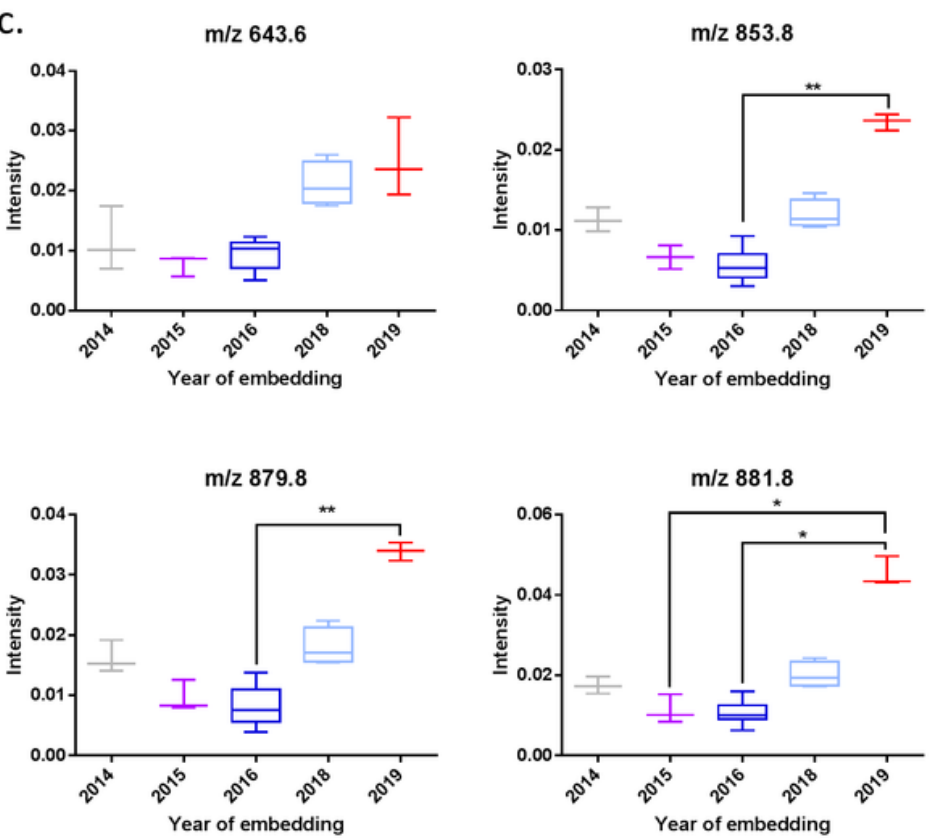

\section{Figure 8}

Effects of conservation on the MS spectra acquired from Leiomyosarcoma samples. A) SpiderMass representation spectra (of 10 laser shots) acquired from the samples preserved since 2014, 2015 and 2016. The spectra have been scaled to 5.87 e4 intensity. We can clearly observe the intensity drop in the mass range between 600-1000 for the years 2014 and 2015 compared to 2019 B) Six-class PCA visualization model built from different time points of Leiomyosarcoma samples (from 2014 to 2019 ). The PC 1 describes 62.27\% variance; PC 2 describes 12.47\% and PC 3 11.29\%. There is a very clear separation by PC 1 between the 2019 and bundle at 2014, 2015 and 2016. C) Relative intensity boxplots of the selected peaks showing the highest contribution to the variance of PC1. The intensities were normalized to the TIC and represented as boxplots for each year with Tukey method whisker definition. There is a significant difference in most of the selected ions but m/z 643.6 between the year 2016 and 2019. * $=p$ value $\leq 0.05, * *=p$ value $\leq 0.01$

\section{Supplementary Files}


This is a list of supplementary files associated with this preprint. Click to download.

- SupplementaryMaterial.docx

- Video1.mp4

- Video2.mp4 